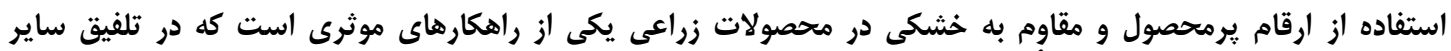

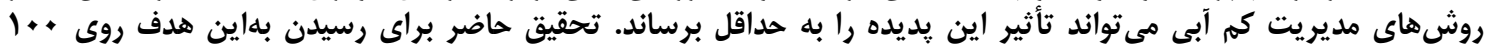

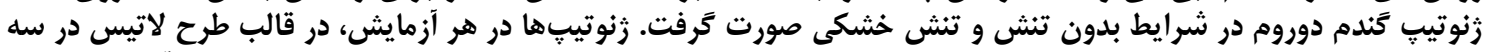

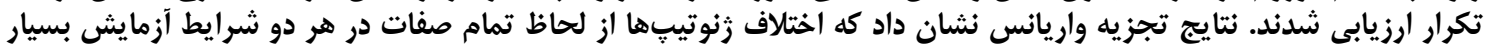

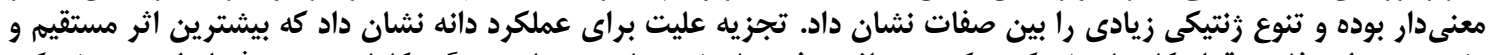

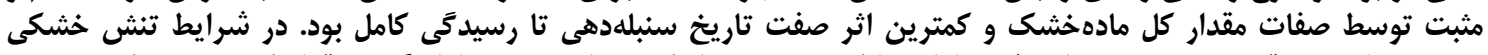

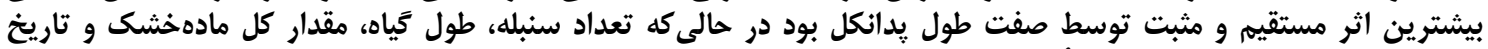

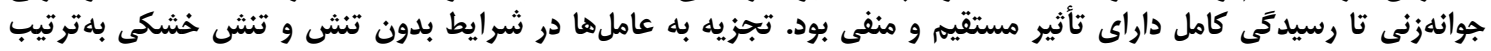

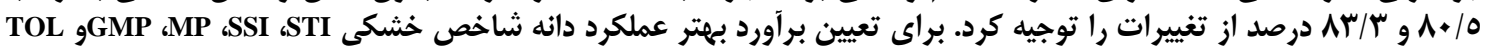

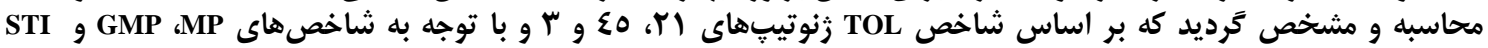

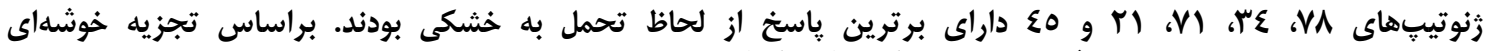

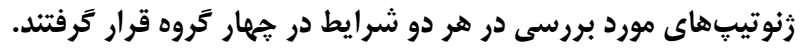

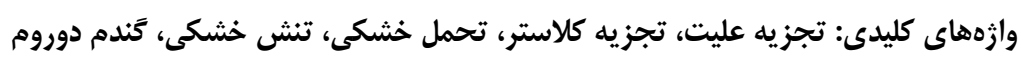

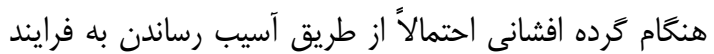

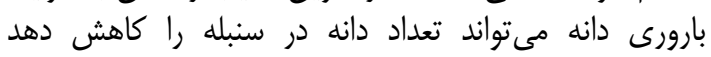

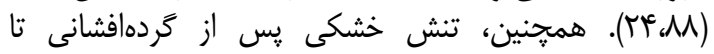

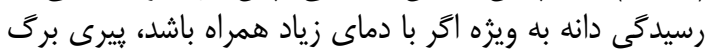

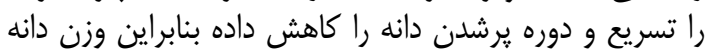

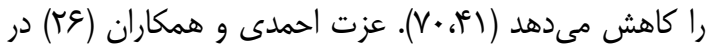

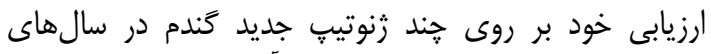

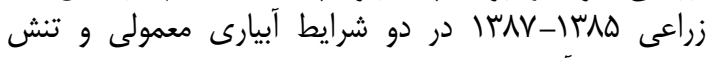

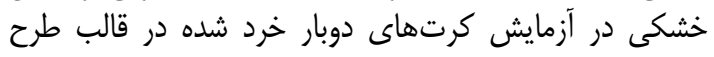

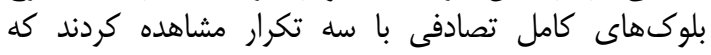

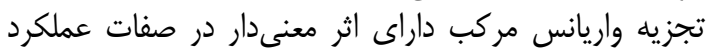

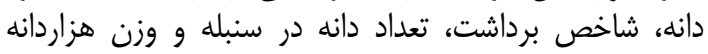

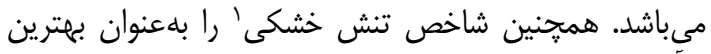

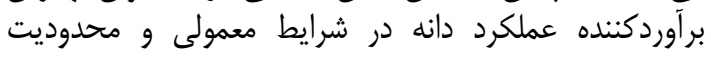

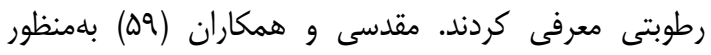

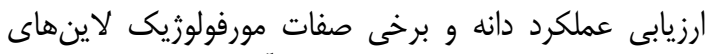

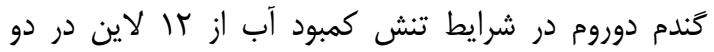

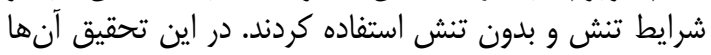

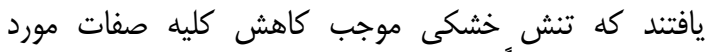

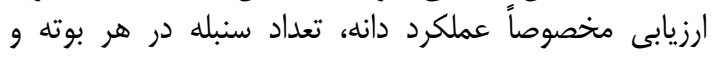

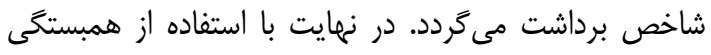

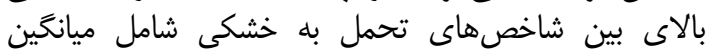

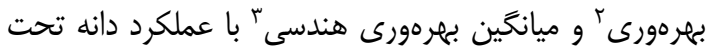

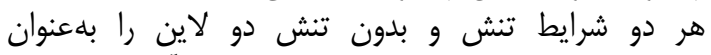

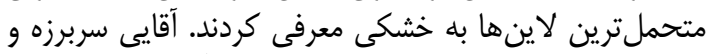

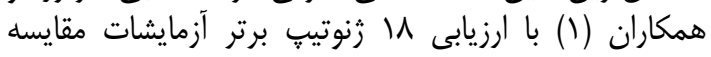

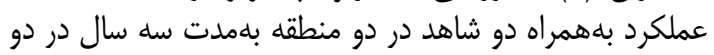

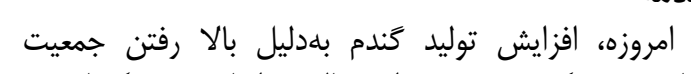

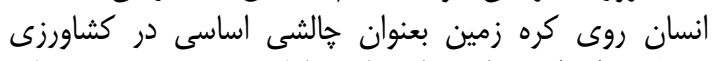

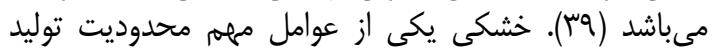

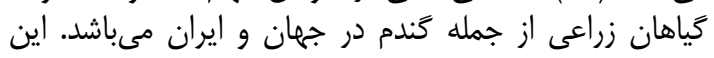

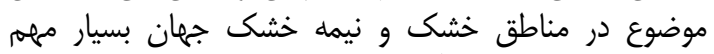

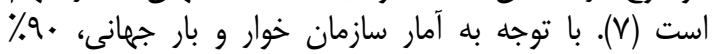

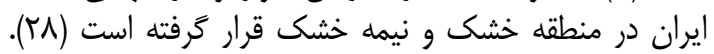

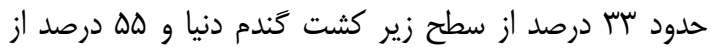

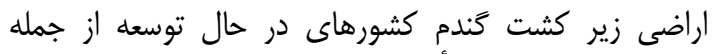

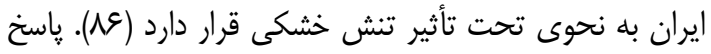

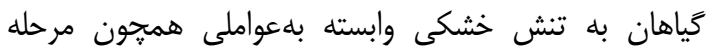

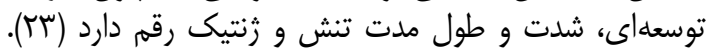

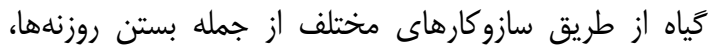

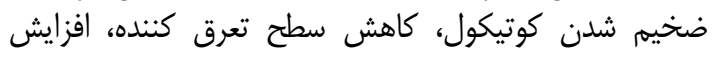

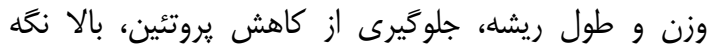

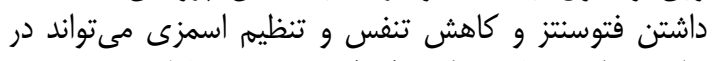

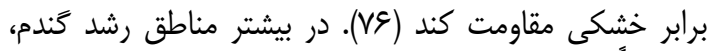

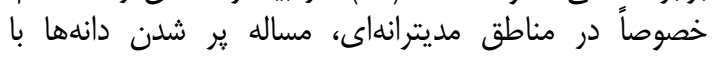

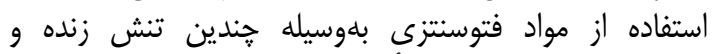

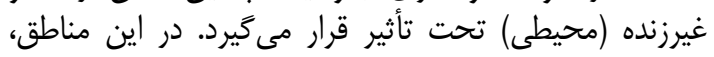

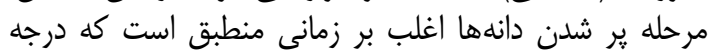

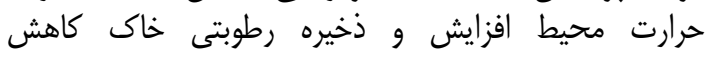

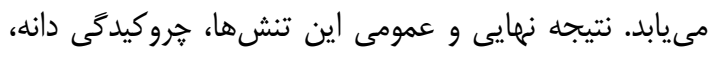

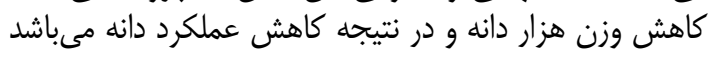

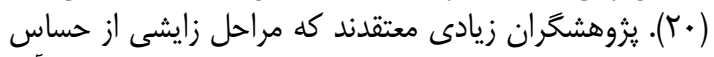

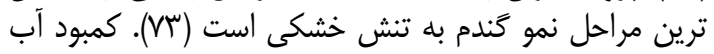




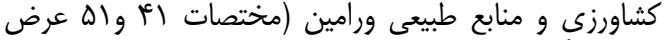

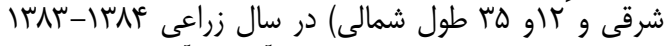

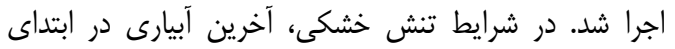

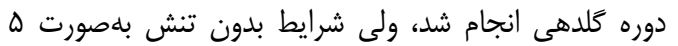

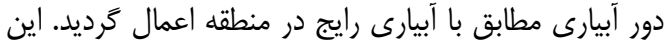

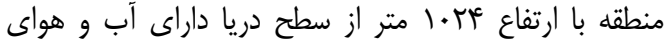

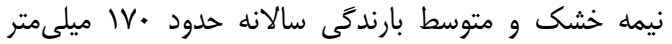

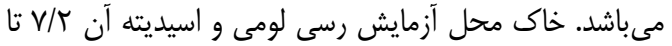
فاصله

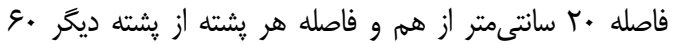

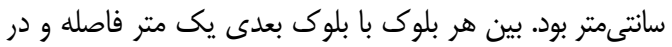

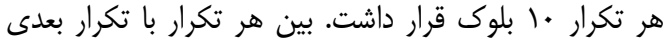

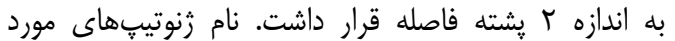

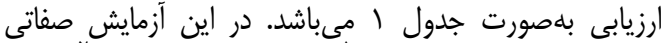

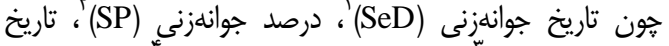

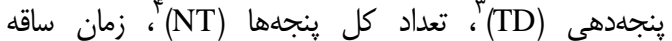

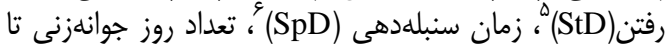

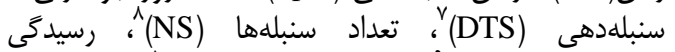

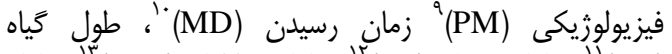
(PH)

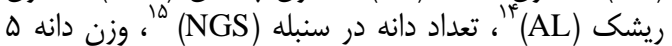
سنبله (SGW) بر (100 GW)

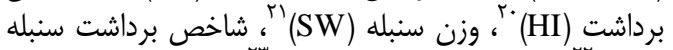
(SHI)

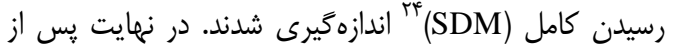

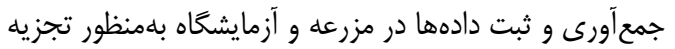

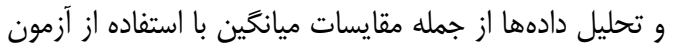

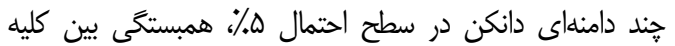

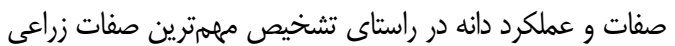

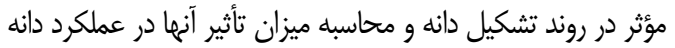

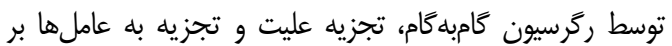

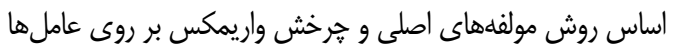

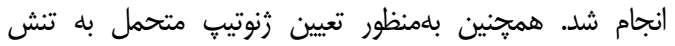
خشكى از شاخصهاى تحمل به خشكى نظير MPP و SSI.STI

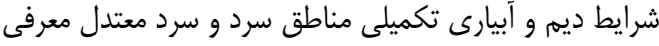

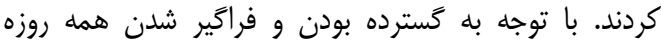

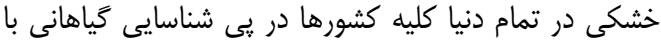

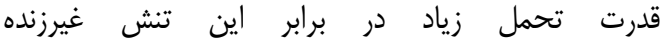

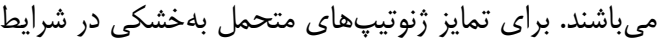

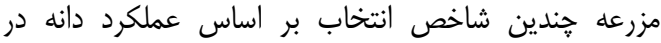

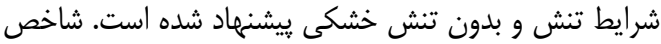

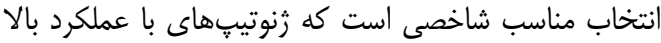

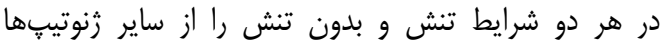

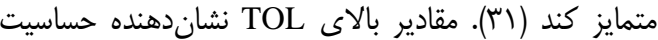

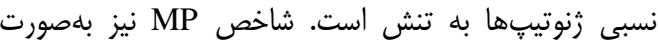

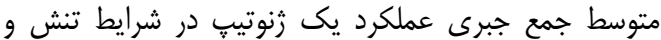

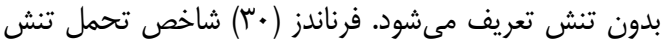

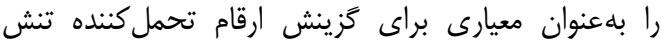

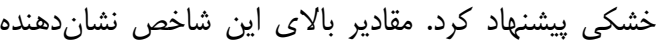

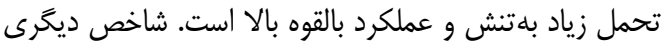

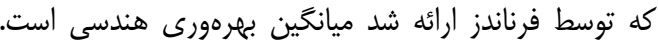

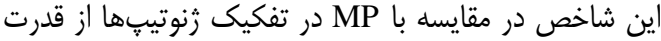

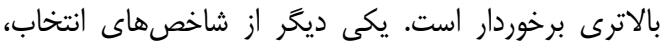

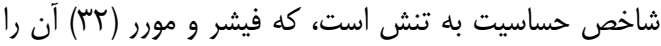

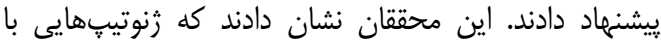

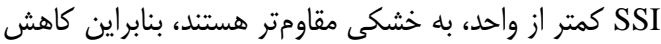

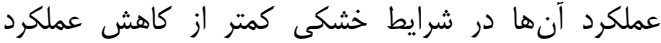

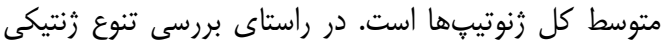

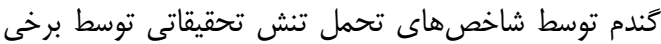
از محققين انجام شده است (1)، (19).

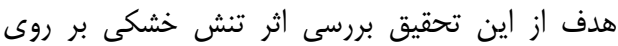

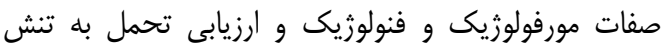
خشكى گَندمهاى دوروم و انتخاب برترين زنوتيكها

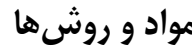

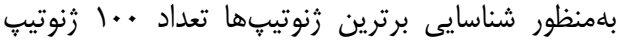

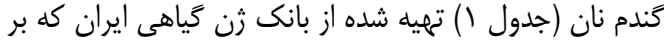

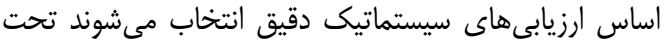

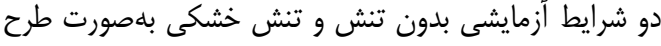

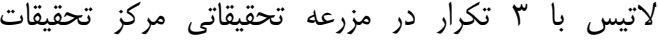

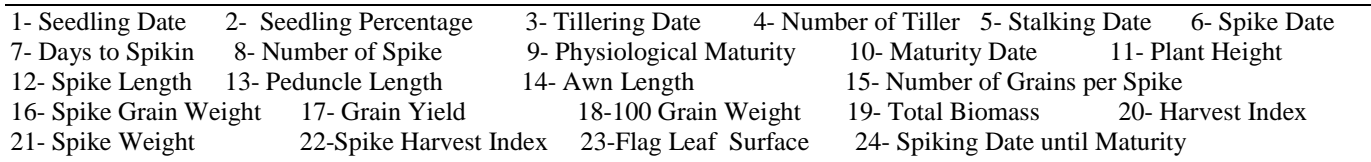


Table 1. Information of under studied wheat genotypes

\begin{tabular}{|c|c|c|c|c|c|c|c|c|c|c|c|}
\hline منشاء & رنوتيّ & شماره & منشاء & رنوتيٍّ & شماره & منشاء & رنوتيٍّ & شماره & منشاء & رنوتيب & شماره \\
\hline نامشخص & IfYY & VE & ايران & $F F \cdot V$ & Q1 & ايران & rQVA & rq & ايران & $r \cdot r r$ & 1 \\
\hline ايران & five & V & ايران & $r \cdot \Delta r$ & $\Delta T$ & ايران & FTOI & $T V$ & ايران & r\&AF & $r$ \\
\hline ايران & rVYq & vı & ايران & 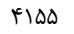 & $\Delta \Gamma$ & ايران & rه人 & $r \Lambda$ & نامشخص & 191. & r \\
\hline ايران & Five & va & ايران & FYA & $\Delta f$ & ايران & 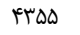 & rq & ايران & IVIS & f \\
\hline ايران & ודזץ & ᄉ. & ايران & Frife & $\Delta \Delta$ & ايران & FYAT & r. & ايران & rris & $\Delta$ \\
\hline ايران & $r \cdot \Delta S$ & 11 & يرتغال & $\Delta \cdot V \Lambda$ & $\Delta S$ & نامشخص & 1991 & ו & ايران & Frtq & 8 \\
\hline نامشخص & r919 & Ar & ايران & FYID & $\Delta V$ & نامشخص & 199. & Tr & ايران & & v \\
\hline ايران & $I F M$ & $\Lambda \mu$ & ايران & $r \Delta \wedge 1$ & $\Delta \wedge$ & مكزيك & سفيد & זץ & ايران & $f \cdot \Delta S$ & $\Lambda$ \\
\hline ايران & سردارى & ᄉf & ايران & $r+1$. & $\Delta q$ & ايران & rivg & MF & ايران & TDST & 9 \\
\hline ايران & $r V \Psi V$ & $\Lambda \Delta$ & ايران & FTV & 4. & ايران & FITV & TL & مكزيك & reto & 1. \\
\hline ايران & ATM & N & ايران & אזאו & 8) & ايران & rIVI & קצ & نامشخص & 1911 & 11 \\
\hline ايران & וצו & NV & ايران & FYFE & $g r$ & ايران & FETE & rV & ايران & TQMF & IT \\
\hline ايران & Tr.r & $M$ & ايران & reve & gr & آرزانتين & QHT. & r & ايران & $1 V \cdot 1$ & זו \\
\hline نامشخص & & 19 & نامشخص & ITSAF & at & ايران & $r \backslash \Delta \Delta$ & rq & نامشخص & MFTh & $1 f^{f}$ \\
\hline مكزيك & TNAT & q. & ايران & 1199 & $9 \Delta$ & نامشخص & 19V0 & f. & ايران & $r .11$ & 10 \\
\hline ايران & rI\$A & 91 & ايران & TLTH & 99 & ايران & תTri & i) & ايران & & 19 \\
\hline ايران & 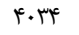 & QT & نامشخص & Ifrr & gV & نامشخص & $r \cdot \Delta \Gamma$ & Fr & ايران & $r F . r$ & IV \\
\hline ايران & آذر r & שו & ايران & זחוץ & 91 & استر اليا & QTKq & Tr & ايران & rIDV & M \\
\hline ايران & $r \cdot \lambda r$ & $9 f$ & آرزانتين & DHTY & 99 & نامشخص & $r \wedge \Delta \Delta$ & Fi & ايران & $f f=1$ & 19 \\
\hline ايران & Fryes & 90 & ايران & YIST & v. & نامشخص & $r \Delta \cdot \Delta$ & $i_{0}$ & ايران & q q & $r$. \\
\hline نامشخص & $1 f \cdot$. & 98 & ايران & FTrA & VI & نامشخص & 「人). & 19 & ايران & Dגז & rI \\
\hline ايران & Fry. & qv & ايران & 1419 & vr & نامشخص & 1918 & FV & ايالات متحده & $r \Delta \Delta V$ & r \\
\hline جمهورى جى & $F v \cdot v$ & 91 & ايران & rD\&G & $v^{\mu}$ & ايران & $10 \cdot r$ & is & نامشخص & 1499 & זי \\
\hline ايران & שיז" & 99 & ايران & ץ एव & $v^{e}$ & نامشخص & TA9T & $\mathrm{kq}$ & ايران & Q DFN & re \\
\hline نامشخص & $r \Delta .9$ & $1 .$. & ايران & GES & Va & ايران & erva & Q. & نامشخص & 1910 & to \\
\hline
\end{tabular}

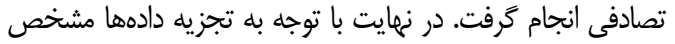

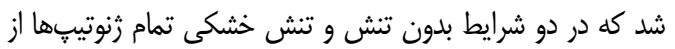

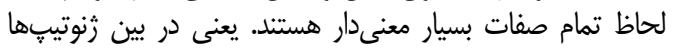

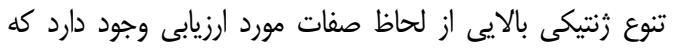

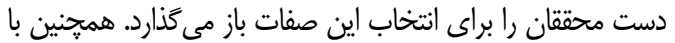

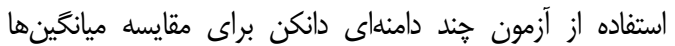

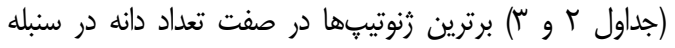

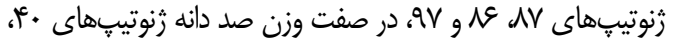

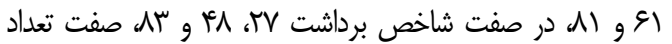

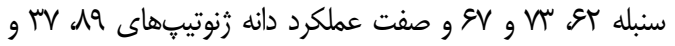

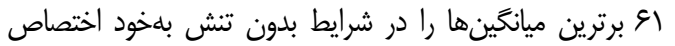

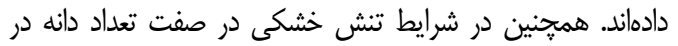

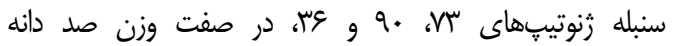

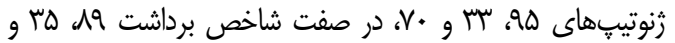

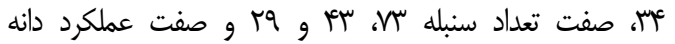

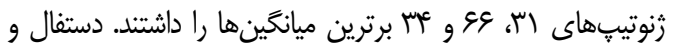

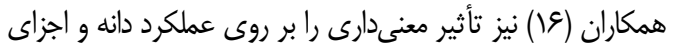

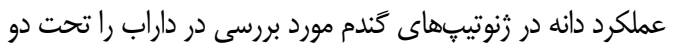

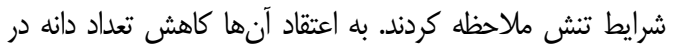

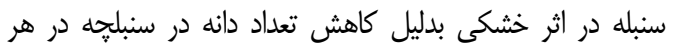

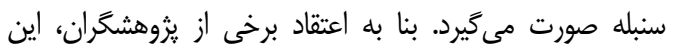

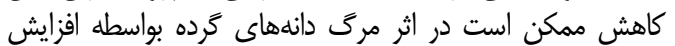

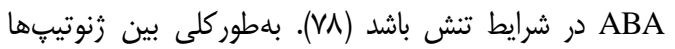

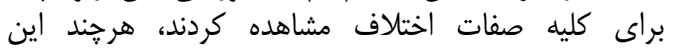
اختلافها براساس تجزيه واريانس براى بران برخى از از آنها معنى دار نبود (عا). در اين مطالعه تنش خشاس خشكى تعداد دانه در
، $M P=\frac{Y_{S}+Y_{P}}{2}$

. $S T I=\frac{Y_{S} \times Y_{P}}{\bar{Y}_{P}^{2}}$

$$
\begin{array}{r}
\text { SSI }=\frac{1-\left(\frac{Y_{S}}{Y_{P}}\right)}{1-\left(\frac{\bar{Y}_{S}}{\bar{Y}_{P}}\right)} \\
\text { GMP }=\sqrt{\left(Y_{S} \times Y_{P}\right)} \\
T O L=Y_{P}-Y_{S}
\end{array}
$$

همبستخى بين شاخصهاى فوق نيز محاسبه و نمودارهاى

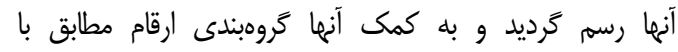

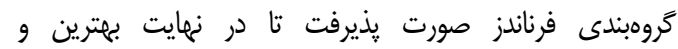

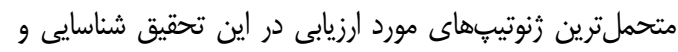

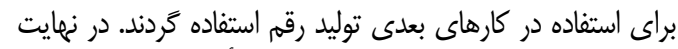

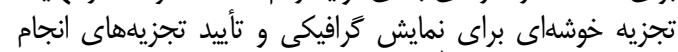

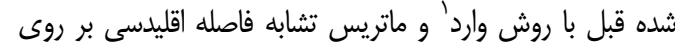

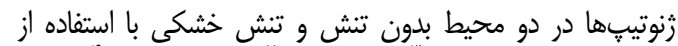

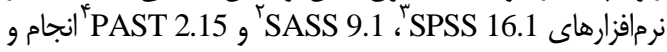
محاسبه گَرديد.

نتايج و بحث

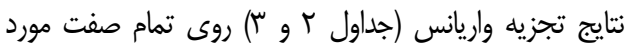

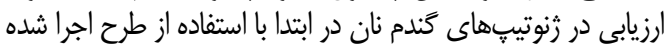

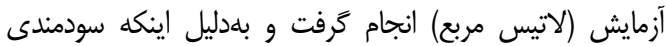

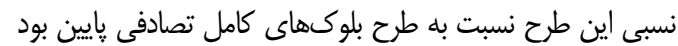

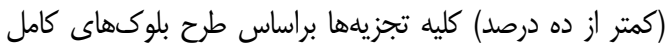


سنبلهدهى، تعداد روز جوانهزنى تا سنبلهدهى، طول ريشك و

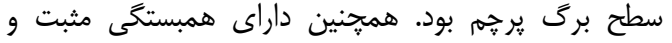

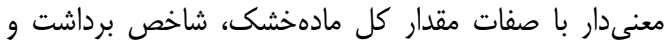

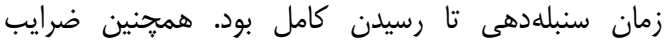

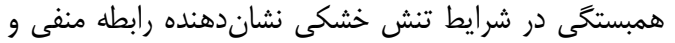

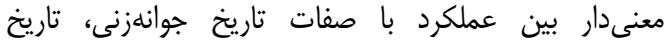

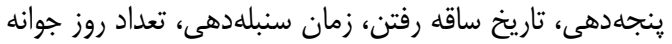

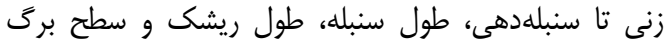

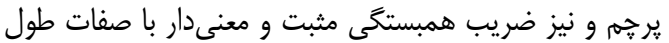

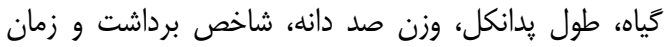

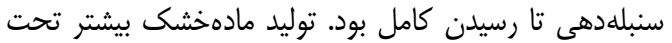
شرايط تنش خشكى بهخصوص در در طول دوره ير برد شدن دانه

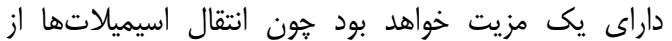

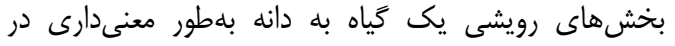

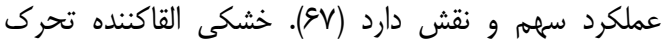

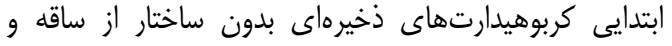

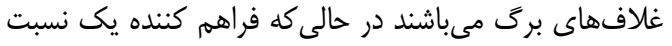

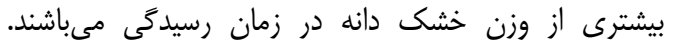

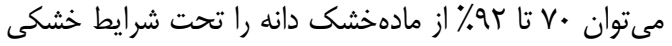

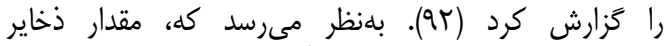

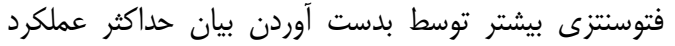

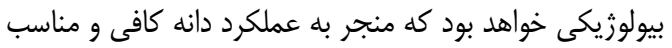

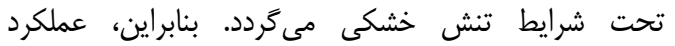

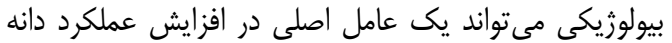

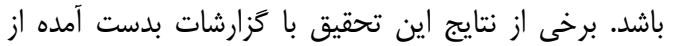

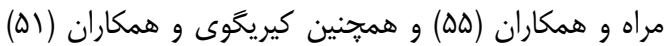
مطابقت داشت.
سنبله را در كليه زنوتيڤها تحت تأثير قرار داد كه اين موضوع

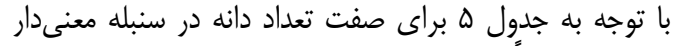

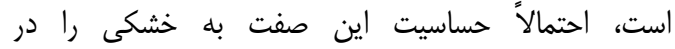

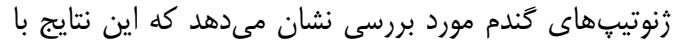

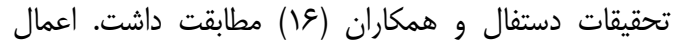

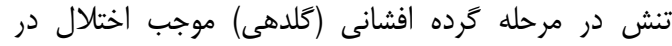

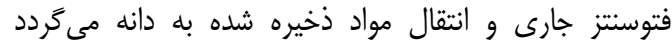

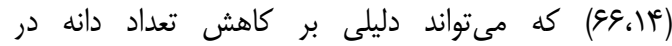

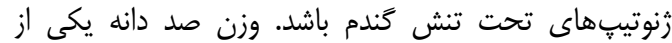

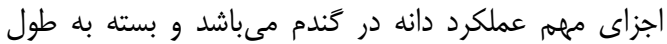

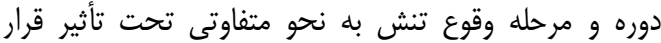

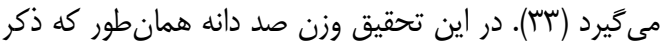

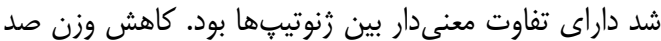

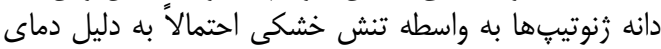

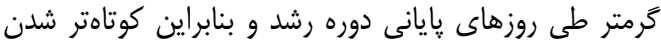

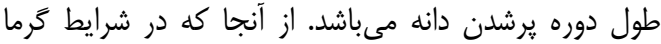

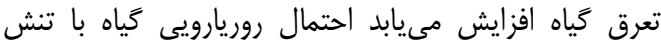

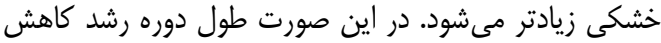

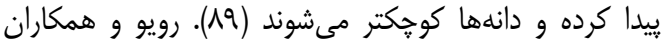

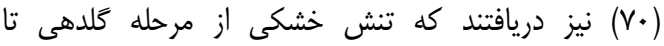

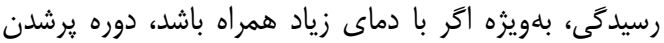

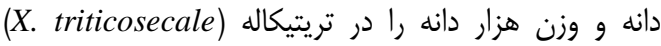

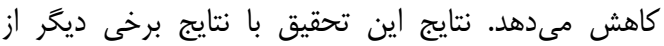

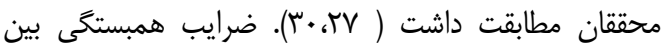
صفات ارزيابى شده در شرايط بدون دان تنش نشان نشان دهند

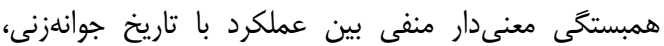

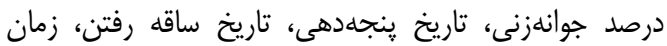


Table 2. Analysis of variance of traits under non-stress condition

جلول r- تجزيه واريانس صفات در شرايط بدون تنش

\begin{tabular}{|c|c|c|c|c|c|c|c|c|c|c|c|c|c|}
\hline \multicolumn{12}{|c|}{ (ميانكين مربعات) MS } & (درجه آزادى)df & منابع تغييرات \\
\hline $\mathrm{SL}$ & PH & MD & PM & NS & DTS & $\mathrm{SpD}$ & StD & NT & TD & SP & $\mathrm{SeD}$ & & صفات \\
\hline $1 / 1.9^{\text {s.th }}$ & $\| /\left.\Delta r\right|^{*}$ &.$|\Delta| f^{*}$ & . & D.MTI & $\cdot / 099^{\text {sulat }}$ & 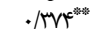 &.$/ 19 *$ &.$/ 1 \wedge q^{*}$ & 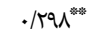 &.$/ 11 e^{w a t a s}$ & . $/$ Y S & 9 & 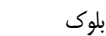 \\
\hline$r / / . . r^{n \cdot m}$ & EFT/q. D. & $S 4 / N G V^{*}$ & $\Delta Q / N G /$ & $\cdot|9 \lambda|^{a b}$ & 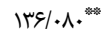 & $1 \mathrm{Vr} / 99 V^{*}$ & $11 \cdot / 1 r^{*}$ & $\Delta / \cdot 19^{* \omega *}$ & $n /$ raqua & $\mathrm{VN} / \mathrm{rNV}^{*}$ & $\Gamma / \Delta \cdot \Gamma^{\prime}$ & 99 & تيمار \\
\hline.$/ 9 M F$ & $9 / \Delta) \&$ & $\cdot / r \cdot V$ & 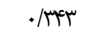 & $\cdot / \pi T 1$ & $\cdot|\Delta \Delta|$ &.$/ T \Delta q$ & . Trq & $\cdot / \triangle \& \Delta$ & 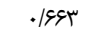 & . THAF &.$/ \pi r$ & 191 & خطا \\
\hline SDM & FLA & SHI & SW & $\mathrm{HI}$ & $\mathrm{TB}$ & 100GR & GY & SGW & NGS & $\mathrm{AL}$ & $\mathrm{PeL}$ & & صفات \\
\hline $1 / \pi 94^{m}$ & $\mathrm{r} / \bar{q} \gamma^{\text {wa* }}$ &.$/ . r^{\text {math }}$ & $.1 .19^{* * *}$ &.$|. \cdot|^{* \infty}$ & $1 / 918^{\text {sats }}$ & $\cdot|\cdot|^{* \ldots+\infty}$ & 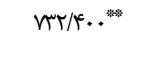 & $\cdot|\cdot \cdot|^{\text {sis }}$ & $\cdot /$ M $\left.\right|^{*}$ & $.1999^{*}$ & 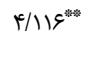 & 9 & بلوك \\
\hline 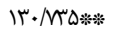 & 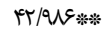 & $\cdot / \Gamma \varphi_{\text {絭 }}$ & ./19Q*** & $\cdot / .1 f_{*}^{*}$ & $F \Delta / T V \cdot \% *$ & 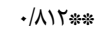 & 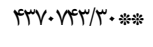 & $9 / 99) \approx$ & $19 / \Delta \mathrm{QV} /$ *** & 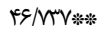 & 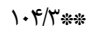 & 99 & تيمار \\
\hline$\cdot(\mathrm{N})$ & $T / K r$. &.$/ .+4$ &.$/ \cdot 1 \mathrm{~V}$ &.$/ .+F$ & $1 / \cdot 11$ &.$/ . .1$ & $198 / 1 .$. &.$/ .4$ & . & . / TqV & $r / \Lambda \cdot \omega$ & 191 & خطا \\
\hline
\end{tabular}

Table 3. Analysis of Variance of traits under stress condition

جدول س- تجزيه واريانس صفات در شرايط تنش خشكى

\begin{tabular}{|c|c|c|c|c|c|c|c|c|c|c|c|c|c|}
\hline \multicolumn{12}{|c|}{ (ميانكين مربعات) MS } & \multirow[t]{2}{*}{$\begin{array}{l}\text { آزادى) } \\
\text { آزئ) df }\end{array}$} & \multirow{2}{*}{ منابع تنييرات } \\
\hline SL & PH & MD & PM & NS & DTS & SpD & StD & NT & TD & SP & $\mathrm{SeD}$ & & \\
\hline$\cdot / 24 M^{-\infty}$ & $V / r Q Q^{\circ \circ}$ & $\cdot / T V T=$ & 每/ & $1 / T /{ }^{\infty}$ & $\cdot / 100^{\circ 0}$ & • & $\cdot / 1999^{\circ 00}$ & . /q. & $\cdot / \% 9 \Lambda^{\circ \infty}$ &.$/ T I V^{\circ}$ & . $/$ M & 9 & بلوك \\
\hline $\mid V / T I T^{+\infty}$ & $4 \pi T / 919^{\circ 0}$ & $\tau \cdot 1 \cdot r \omega^{\text {on }}$ & rq/991 & 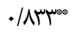 & $\mid Q T / A V Q^{\circ \circ}$ & $194 / \Delta T T^{n o s}$ & 1. NASE & r/ץ.๘ & $n / r<990$ & $|V / 9 Y|^{100}$ & $r / \Delta \cdot r+\infty$ & 99 & تيمار \\
\hline$\cdot / N 1$ & $0 / 819$ & . & $\cdot|r \Delta|$ &.$\left.|9|\right|^{f}$ &.$/ 9 \cdot 0$ &.$/ F T$ & • & $\cdot / N A$ & . $199 \pi$ & T & . & 191 & خطا \\
\hline SDM & FLA & SHI & SW & $\mathrm{HI}$ & $\mathrm{TB}$ & 100GR & GY & SGW & NGS & $\mathrm{AL}$ & PeL & & صفات \\
\hline$\cdot / \Lambda 19^{+\infty}$ & $\Delta / / \lambda e^{\infty e o s}$ & . & . $/ .+c^{+\infty o \infty}$ & . & $r / T r q^{\circ 0}$ & $.1 . .11^{\infty \infty}$ & $\mid 9941 / 4 \ldots$ & $\cdot / . .11^{\infty}$ & $\cdot / r Y^{\text {cos }}$ & $\cdot / \pi r \cdot a$ & १/१९q४० & 9 & بلوك \\
\hline | & TNTOV & $\cdot / \cdot 11^{\infty}$ & .1 .9400 &.$/ .19^{\circ \infty}$ & 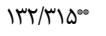 & $\left.\cdot|\Delta|\right|^{100}$ & MFIDIT/N" & $r / 9911^{\circ \infty}$ & $1 \% / r 99^{\circ 0}$ & $r \Delta / \Delta A^{* \infty}$ & $11 / \mu \Gamma^{\text {No }}$ & 99 & تيمار \\
\hline$\cdot|\Lambda|$ & $\Delta / F M$ & $\%$. r & $\% 1$ & $\%$. r & $r / \Lambda \cdot \Lambda$ &.$/ .1$ & |fges/N.. &.$/ . r$ &. $\mid+91$ & $. / 49 \mid$ & g/rVT & 191 & خطا \\
\hline
\end{tabular}



ارزيابى زنوتيڤهاى گندم دوروم در شرايط آبيارى و تنش خشكى

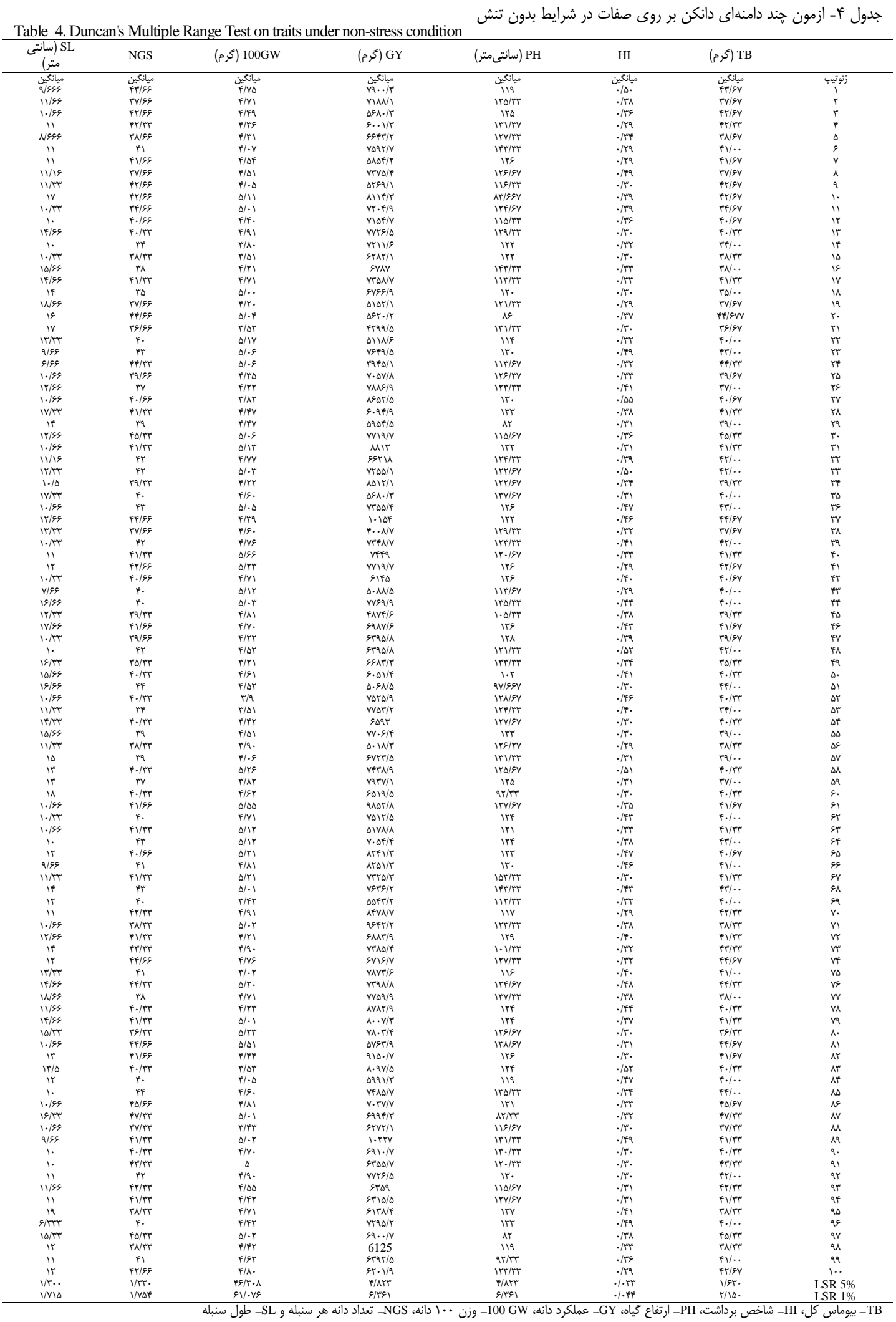



يخوهشنامه اصلاح گياهان زراعى / سال نهم/ شماره ع / زمستان عهبا

Table 5. Duncan's Multiple Range Test on traits under stress condition

جدول ه- أزمون گند دامنهاى دانكن بر روى صفات در شرايط تنش خشكي

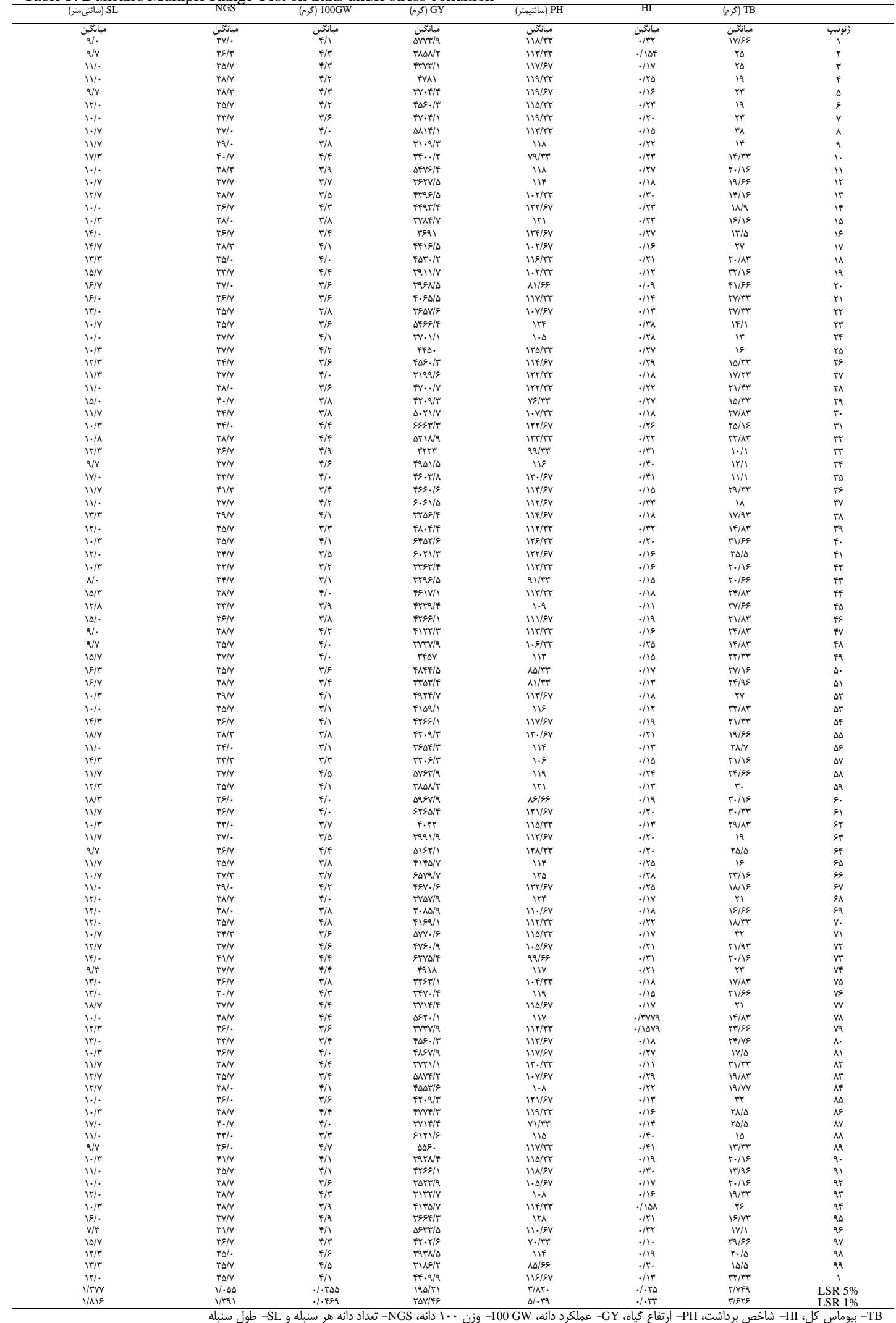




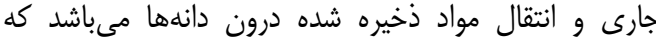

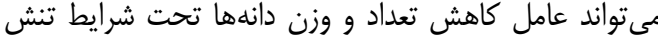

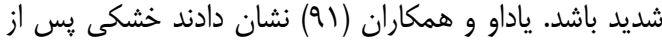

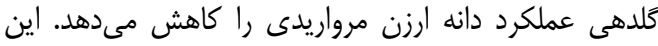

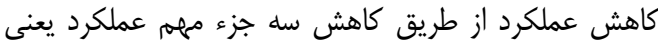

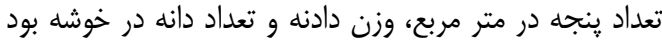

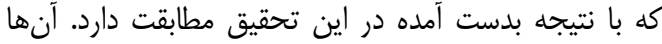

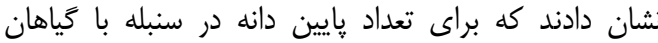

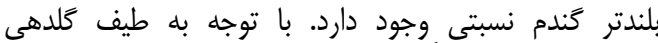

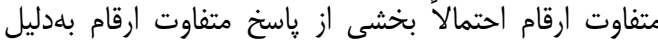

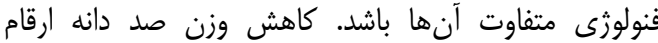
مختلف به تنش خشكى نشانداندانداه عدام تامين فتوسنتزى

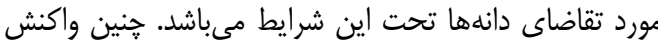

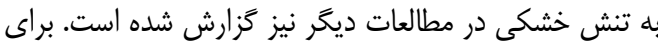

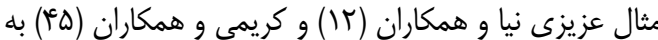

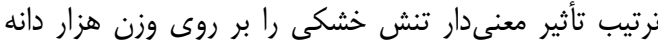

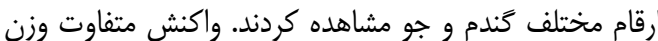

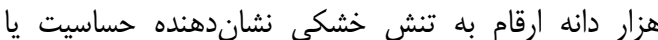

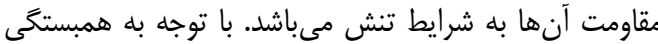

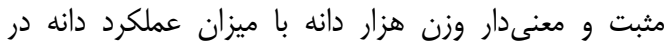

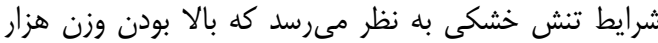

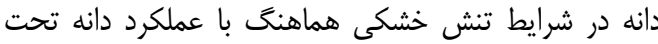

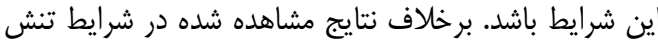

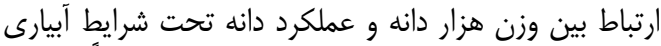

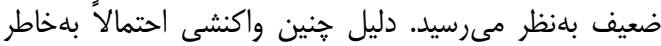

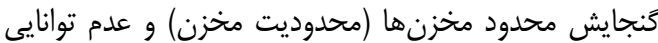

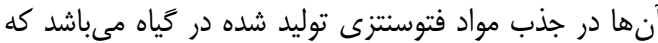

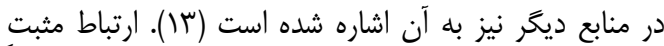

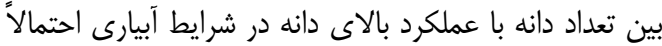

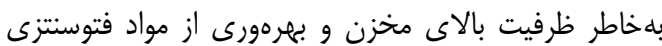

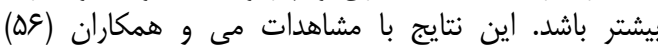

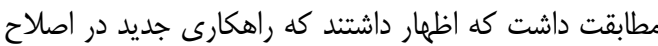

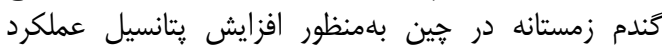

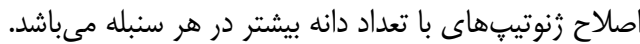

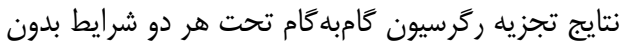

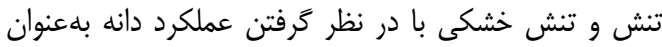

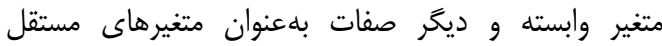

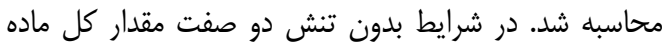

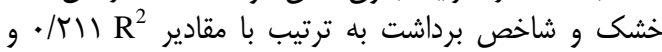

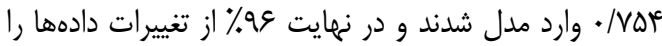

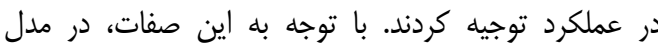

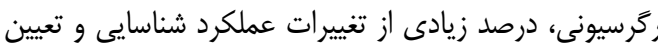

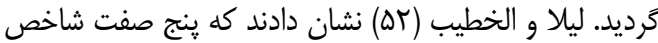

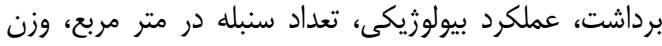

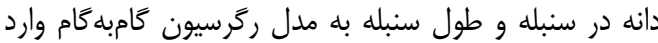

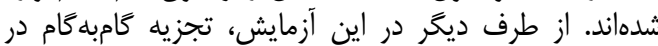

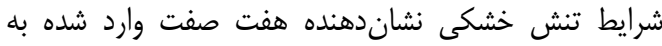

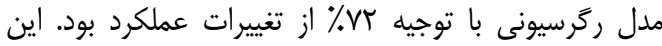

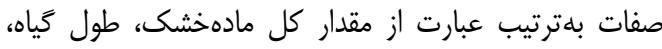

همبستخى فنوتيبى ميان صفات مختلف در هر دو شرايط

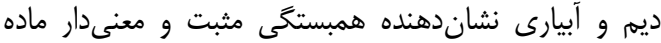

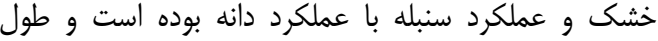

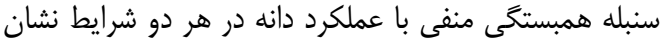

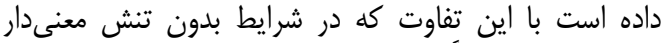

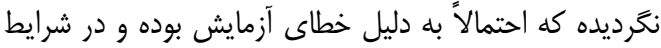

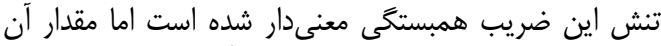

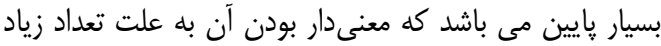

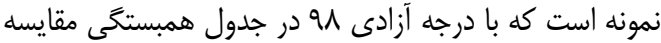

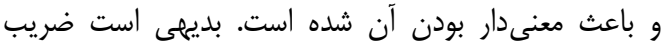

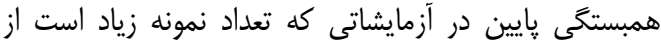

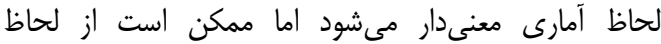

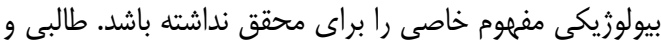

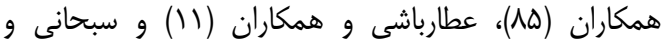

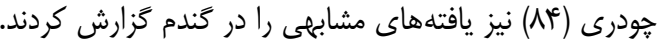

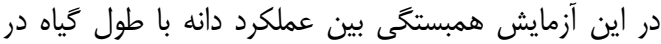

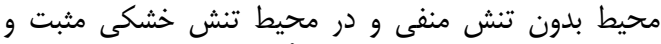

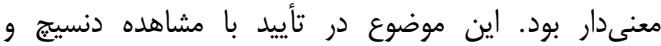

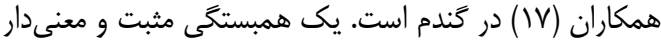

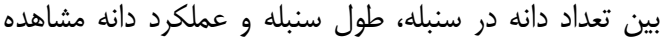

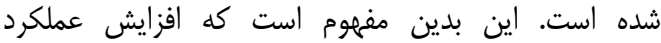

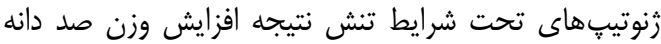

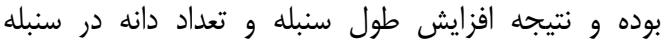

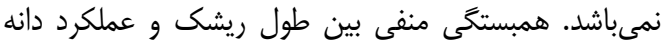

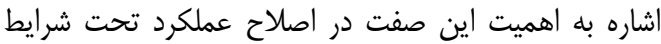

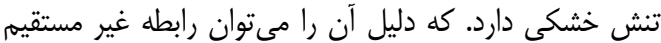

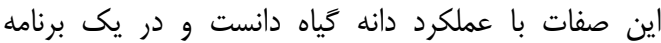

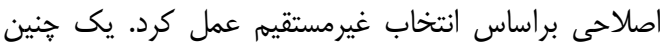
ارتباطى ناشى از قدرت كياهان در اندان انجام فتوسنتز بواسطه

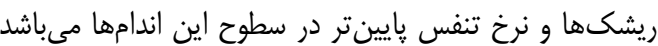

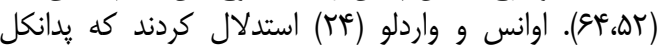

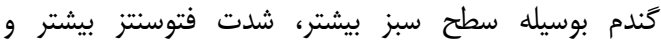

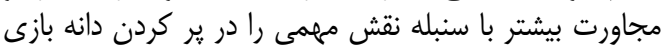

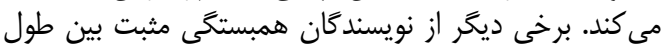

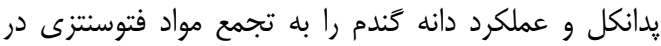

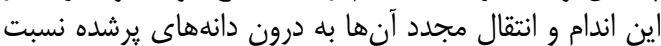

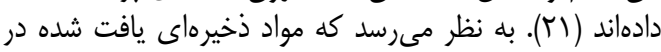

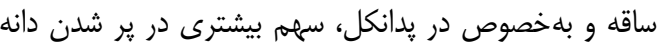

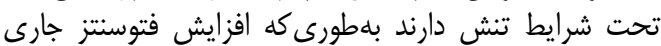

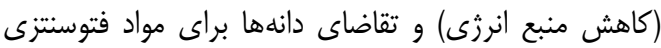

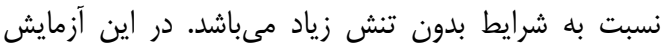

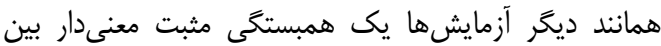

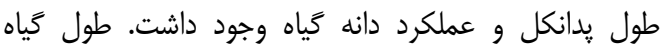

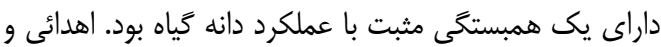

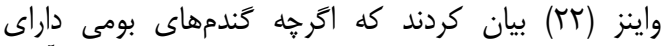

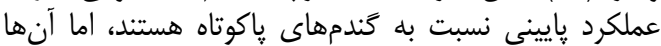

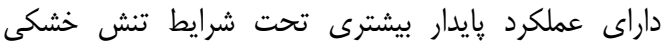

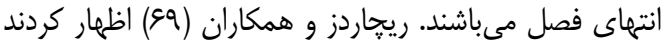

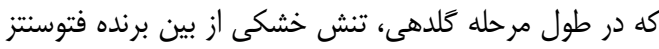


بيشترين اثر غيرمستقيم و منفى توسط شاخص برداشت از

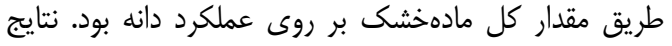

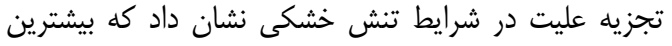

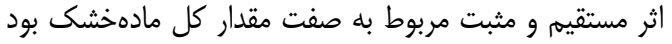

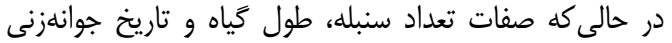

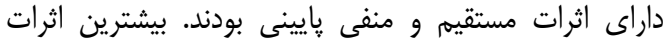

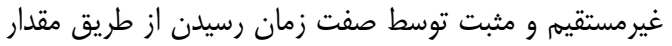

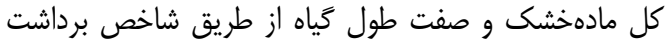

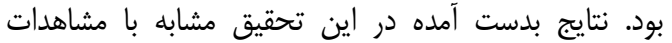

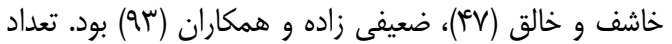

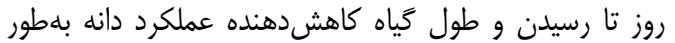

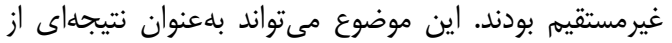

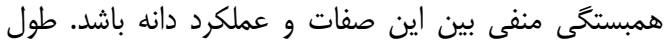

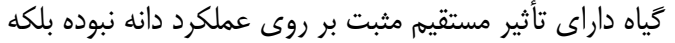

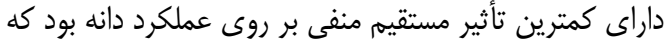

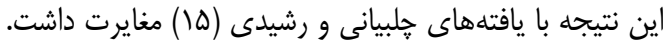

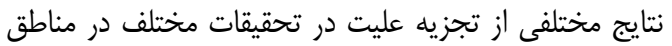

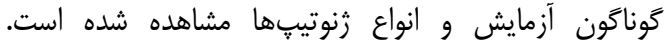

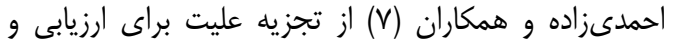

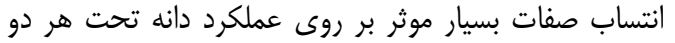

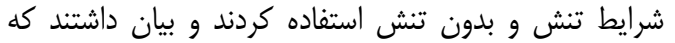

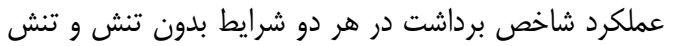

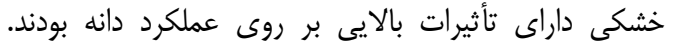

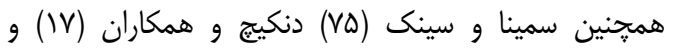

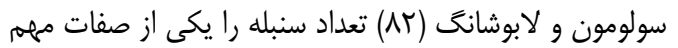

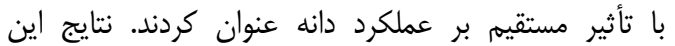

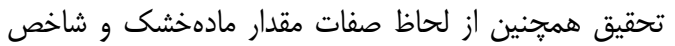

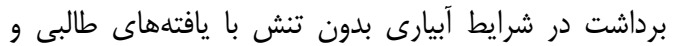
همكاران (Nه) مطابقت داشت.

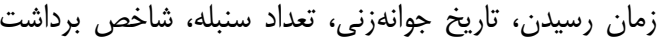

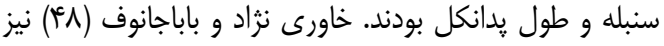

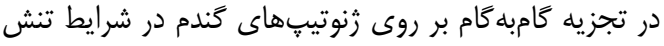

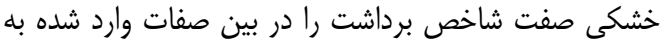

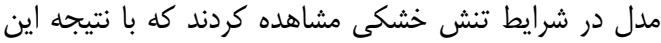

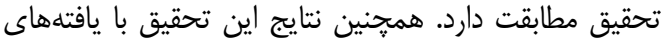

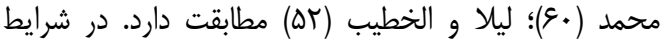

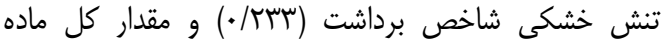

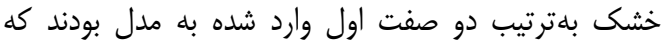

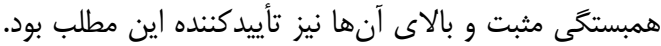

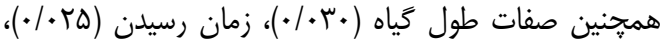

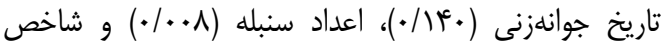

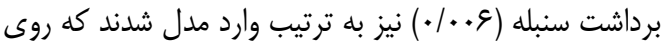

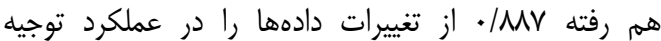

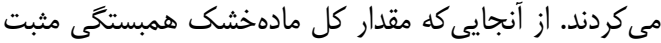

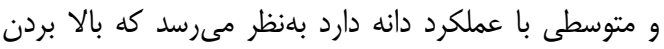

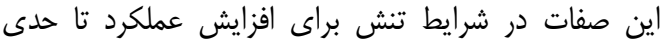

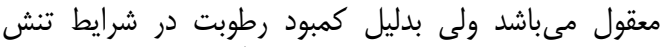
خشكى در مراحل يرشدن دانه عملكرد آن در مقايسه با شائ شايط

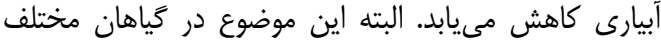

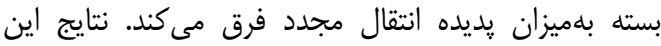

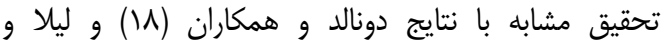

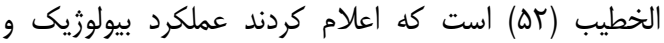

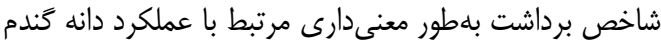

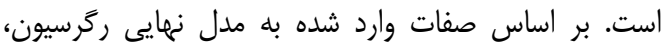

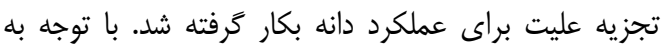

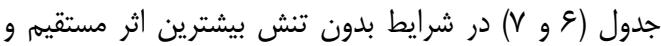

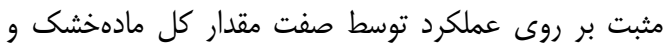
كمترين اثر توسط صفت شاخص توسط برداشت بود. همجنين مادهن

Table 6. Path analysis under non-stress condition

جدول و- تجزيه عليت در محيط بدون تنش

\begin{tabular}{|c|c|c|c|c|}
\hline كل & HI & TB & اثرات غير مستقيم & صفات \\
\hline$\cdot / 4 \cdot$ & $-\cdot / \mathrm{V} \cdot 1$ & - & $1 / / T V$ & $\mathrm{~TB}$ \\
\hline.$/ \& \Delta \Lambda$ & - & -.1998 & $1 / 1.1$ & $\mathrm{HI}$ \\
\hline
\end{tabular}

Table 7. Path analysis under stress condition

\begin{tabular}{|c|c|c|c|c|c|c|c|c|c|}
\hline كل & SHI & NS & $\mathrm{SeD}$ & MD & PH & TB & HI & / مسنقيم / & صفات \\
\hline$\cdot / 4 \cdot$ &.$- / \cdot \cdot 1$ &.$/ \cdot 1$ &.$/ \cdot{ }^{f}$ &.$- / .1 T$ &.$- / \cdot \cdot 1$ & $. / 9 M M-$ & - & $1 / .94$ & $\mathrm{HI}$ \\
\hline.$/ \& \Delta \Lambda$ & • & $-+\mid \cdot+1$ & 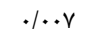 &.$- / \cdot 1 \cdot$ & • & - &.$- / 9 \mathrm{~V} F$ & $1 / 110$ & $\mathrm{~TB}$ \\
\hline$. / 1 F \mid$ & . &.$- \cdot \cdot r$ & $\% v$ & $-1 . \cdot 9$ & - & $-\cdot / \cdot r f$ & . NFA &.$- \mid \cdot .1$ & $\mathrm{PH}$ \\
\hline$-\cdot / \cdot 11$ &.$- / \cdot \cdot 1$ & $-\cdot / \cdot r$ & $-\cdot \cdot \cdot \mu \cdot$ & - & . & $\cdot / \Gamma \cdot \Delta$ & אسץ/.- & צץ./. & MD \\
\hline$-\cdot / T V F^{e}$ & $\cdot$ & $* / .+t^{c}$ & - & /.r. & . & $-\cdot / \mid \Delta \Lambda$ &.$- / 119$ &.$- / . \Delta f^{F}$ & $\mathrm{SeD}$ \\
\hline$-.1 .9 T$ & . & - &.$/ .1$ &.$- / . .9$ &.$- \mid \cdot \cdot 1$ & $.1 . r q$ &.$- / \cdot v f^{f}$ & r & NS \\
\hline.$- / . \cdot 9$ & - &.$- \cdot \cdot r$ & $-\cdot / \cdot \theta \Delta$ &.$- / . r r$ &.$- / \cdot \cdot 1$ &.$/ . \mu F$ & ه ه &.$/ . .1$ & SHI \\
\hline
\end{tabular}


را مشاهده كردند كه با نتايج اين تحقيق مشابه است. سى و

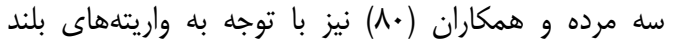

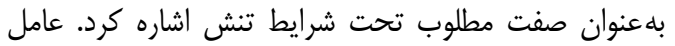

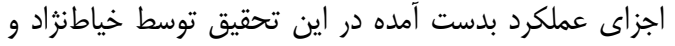

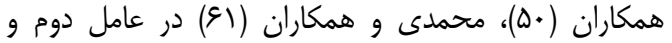

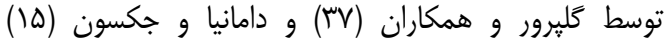

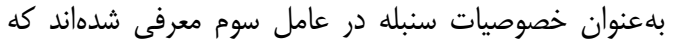

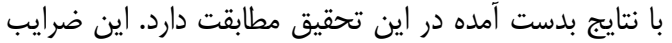

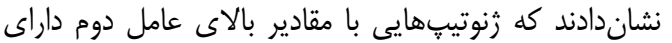

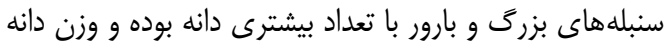

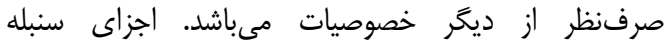
(طول، تعداد سنبله، تعداد سنبلجههاى بارور و و تعداد داد دانه)

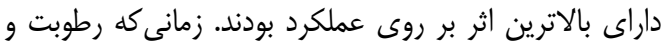

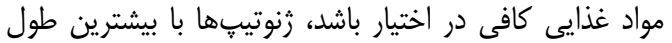

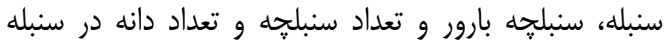

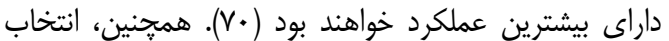

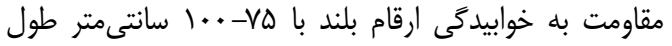

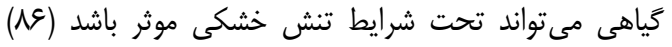

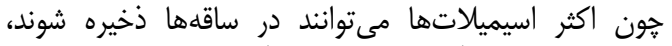

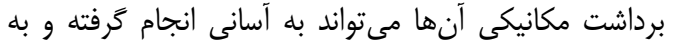

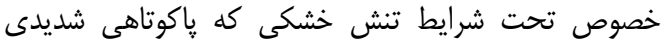

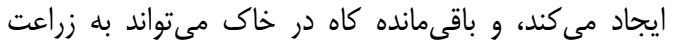

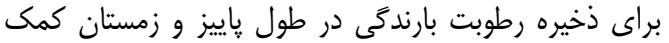

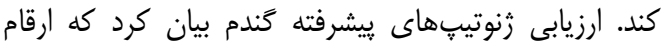

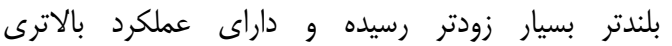

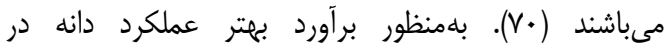

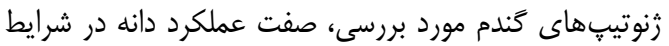

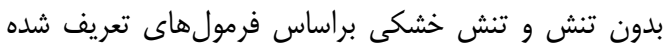

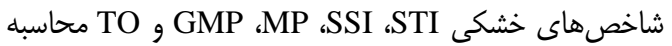
شد. براساس شاخصهاى SOL و

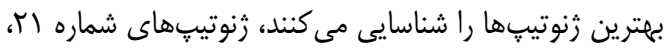

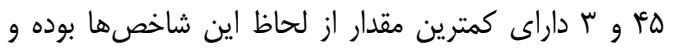

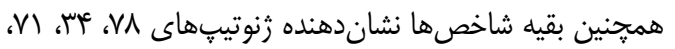

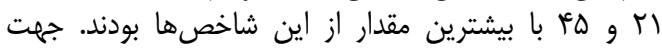

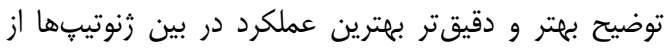

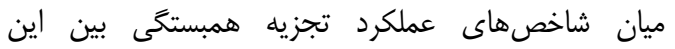
شاخصها انجام گرفت (جدول • (1).
تجزيه بلهعاملها تحت شرايط بدون تنش (جدول ه ه) توسط هفت عامل و تحت شرايط تنش خشك تشكى (جدول

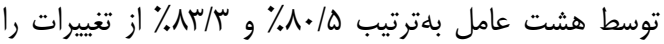

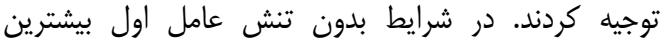

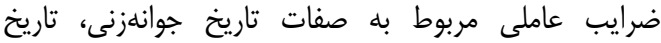

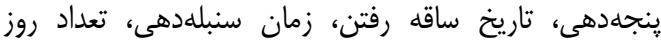

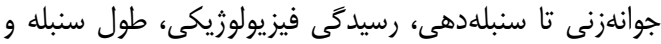

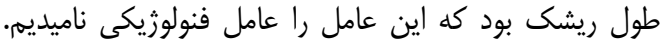

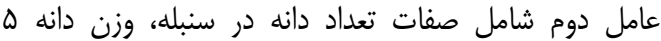
سنبله، وزن صد دانه، وزن سنبله

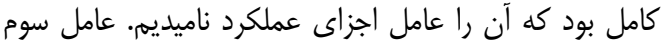

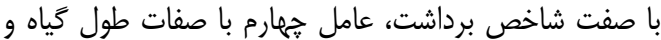

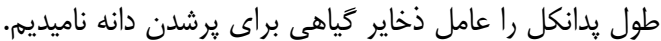

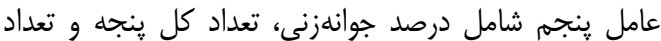
سنبله بود. عامل ششم شامل صفات مقامل دقدار كل مان مادهخشك و

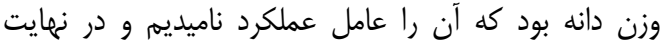

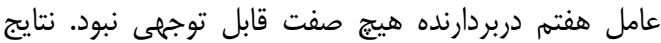

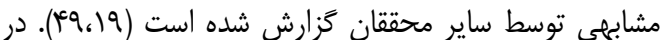

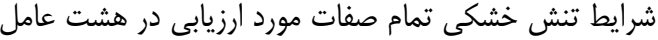

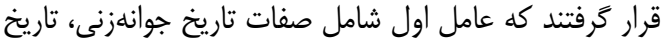

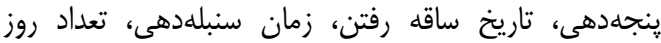

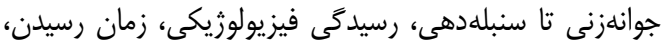

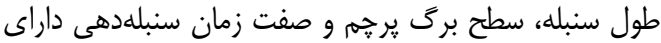

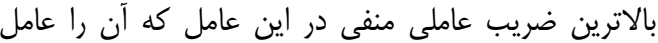

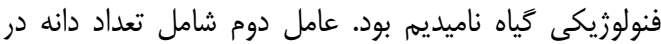

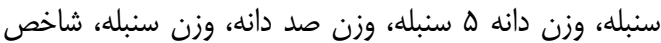

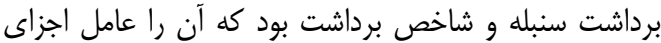

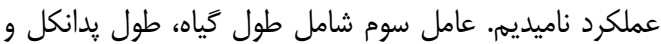

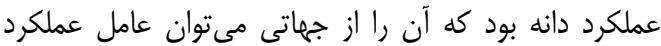

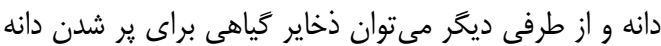

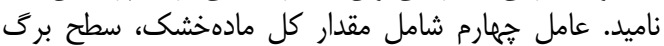

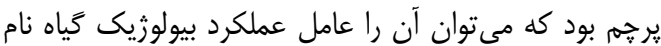

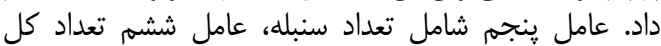

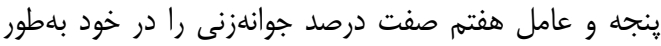

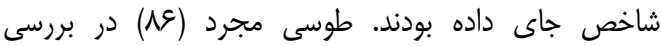

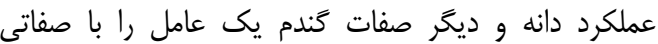

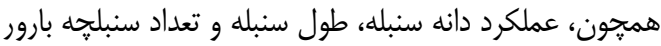


Table 9. Factor analysis for stress condition

جدول q- تجزيه به عاملها در شرايط تنش خشكى

\begin{tabular}{|c|c|c|c|c|c|c|c|c|}
\hline \multicolumn{9}{|c|}{ ضرايب عاملى } \\
\hline$\Lambda$ & $\mathrm{v}$ & 8 & $\Delta$ & $r$ & $r$ & $r$ & 1 & صفات \\
\hline$-.1 .4 v$ & $-.1 .+Q$ & .1 .45 & $-.1 . \wedge f$ &.$/$ Mr & -.1 .48 &.$/ .4$. & $\cdot|\lambda \uparrow|$ & $\mathrm{SeD}$ \\
\hline.$/ M E V$ &.$/ 099$ & $-. / K \mid q$ & -.1 .9 & . MET & $-. / r r \mid$ &.$- / \pi \mu F$ &.$- / K I \Lambda$ & SP \\
\hline 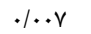 & $.1 .4 t$ &.$- / 199$ & -.1 .9 &.$- / 119$ &.$-|\cdot r|$ &.$/ N Q$ & . /N\&9 & TD \\
\hline ו ו ו & $.1 . .9$ & $\cdot /$ VIf &.$- / \cdot \Delta r$ & $\cdot \mid \cdot v 1$ & $\cdot / 1 \Delta V$ & & -.1 .99 & NT \\
\hline س F./. & $\cdot 1 \cdot 1 \Delta$ &.$/ \cdot 1 f$ & $-\cdot / \cdot \varphi \cdot$ & $-.1 \cdot 1 \mathrm{f}$ & $.1 \cdot v g$ & r.^/./ & . /9T & $\mathrm{StD}$ \\
\hline-.1 .94 & .1110 & |r.|. &.$- / 1 f \Delta$ & - & $\cdot 1 \cdot v^{c}$ & $.1 \cdot 10$ & | ו |q/. & $\mathrm{SpD}$ \\
\hline.$- / T Y V$ &.$/ K A$ & $\%$. & - &.$-|T M|$ &.$/ 1 T V$ &.$- / . \Delta f$ & •/1998 & DTS \\
\hline$-\cdot / \cdot v 1$ & $\cdot /$. & $\cdot \mid \cdot 41$ & $\cdot / 9 \cdot 1$ & . $/ / \Delta T$ & תTr/R & $.1 .99-$ & $-\cdot|| Q \mid$ & NS \\
\hline$\cdot|10|$ & . & THET & 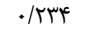 & .1 .19 & . & $\cdot / \cdot \Delta \Delta$ & $\cdot / v \cdot 9$ & PM \\
\hline H & س & 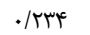 & תזr/ & $.1 .4 \Lambda$ &.$/ F \Delta S$ &.$/ . \Delta g$ & . NTr & $\mathrm{MD}$ \\
\hline.- .1 .11 &.$- / 1 D S$ &.$- / 11$ &.$- / \mu F q$ &.$- / T F \Delta$ &.$/ 498$ &.$- / 119$ &.$- / D F F$ & PH \\
\hline גואו &.$- / I V F$ &.$- / T M M$ &.$- / . \cdot f$ & . &.$- / . \vee$. & .11 .9 & . $/$ NFF $^{\prime}$ & SL \\
\hline ./TTQ &.$- / 191$ & 满 & $-\cdot / \Gamma \& \Delta$ &.$/ 1 r$. &.$/ 4 \wedge$ & $-\cdot /$ TQT &.$- / 4 \wedge 9$ & PeL \\
\hline ./TME & גזו/.- & $-\cdot / r V V$ &.$/ \Delta \mu$ & . /raf & ג גו/.- &.$/ 198$ & . 199$)^{4}$ & AL \\
\hline TK & $-\cdot / F V \Psi$ & $\cdot / T \Delta 1$ & $-\cdot / 111$ &.$- / . .9$ & תזr/. & - /DFA & $-\bullet / 19$. & NGS \\
\hline-.1 .19 & .1 .91 &.$/ N F$ & $-\cdot 11 \cdot 9$ &.$/ 19$ & $\cdot / \cdot \Delta \wedge$ & אזוq/. & גזן & $5 \mathrm{SGW}$ \\
\hline$\cdot|\cdot r|$ &.$/ 114$ & - - /TTV &.$/ 419$ & $-\cdot 1 \cdot \wedge \mathrm{V}$ & r & .1 .09 & | & GY \\
\hline$-.1 . r f$ &.$/ \mu F$. &.$/ . r t$ & $-.1 .+4$ & . & ./499 & $\cdot / V V A$ & $-* / 1 Q$ & $100 \mathrm{GW}$ \\
\hline V V & .11 .9 & 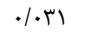 &.$- / \cdot \Delta \Delta$ & $\cdot / 9 \wedge \Delta$ & $\cdot / r \cdot V$ & $-. / 4+1$ &.$/ 1 F 9$ & $\mathrm{~TB}$ \\
\hline$\cdot / r \cdot V$ &.$|+4|$ & $-\cdot / r \cdot V$ & גואו & سקt|-- &.$/ 111$ & GTא/. & سצא/.- & HI \\
\hline . & $\cdot / r \Lambda I$ & . TKE &.$- / 4 \& V$ & . & r..|. & - /\&QV & $-* /$ KE. & SW \\
\hline 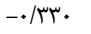 & $-\cdot / K T F^{f}$ & $-.1 .9 \mathrm{~V}$ & $\cdot|F| f$ & תTr/ & .1 .9 & - $/ \& \Delta F$ & .1 .90 & SHI \\
\hline$\cdot / \Delta V V$ & $-\cdot / I V V$ & $\cdot / 1 / 9$ & $\cdot / r \cdot \cdot$ & $\cdot / 1 \Delta S$ & - & $-\cdot / r \cdot \varphi$ & באזא/. & FLA \\
\hline.$/ 199$ & . $/ 1 T V$ & $.1 \cdot v 9$ &.$/ \mu$. & & (1/Rq & $\cdot / \cdot 1$ & . $/$ Vqu & SDM \\
\hline אזגון. & $\cdot / \mathrm{M} M$ & $\cdot \mid V+1$ & . MTK & . MTK & . $/$ DFA &.$/ 494$ & . & واريانس تجمعى \\
\hline $1 / \cdot 11$ & l/fe. & $1 / M+1$ & $1 / 599$ & $1 / V \wedge \Delta$ & $r / .19$ & 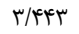 & $V / 998$ & مقدار ويثه \\
\hline
\end{tabular}

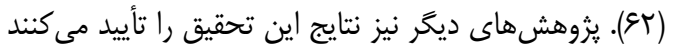

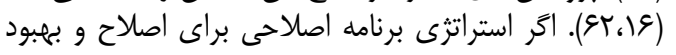

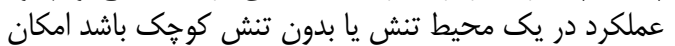

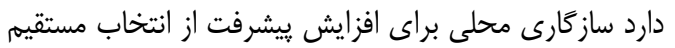

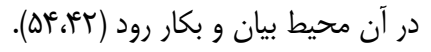

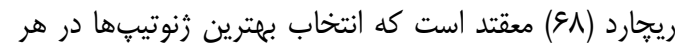

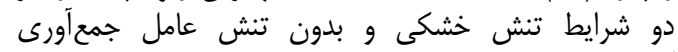

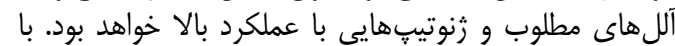

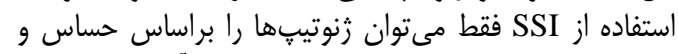

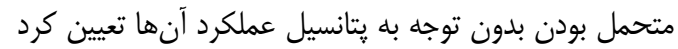

Table 10. Correlation coefficients between drought indices

جدول • ا- ضرايب همبستخى بين شاخصهاى خشكى

\begin{tabular}{|c|c|c|c|c|c|c|}
\hline & Ys & TOL & MP & GMP & SSI & STI \\
\hline Yp & $\left.\cdot|\Delta|^{*}\right|^{* * i n}$ & $.19 \mathrm{~V}^{*} q^{* \ldots}$ & $\cdot / / 9 q^{*}$ & 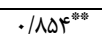 & 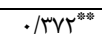 & $\cdot / \Delta \psi^{*} \gamma^{* i=}$ \\
\hline Ys & &.$-|\Gamma \Delta|^{\prime \prime}$ & $\cdot|r \Delta|^{* \prime}$ &.$/ 199^{* * * * *}$ & 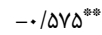 &.$/ 9 \ldots *$ \\
\hline TOL & & & $-1^{* *}$ &.$/ 194^{*}$ & . &.$/ 1199$ \\
\hline MP & & & &.$- / 194^{* 20}$ & - & $-\cdot / 1 / 9$ \\
\hline GMP & & & & &.$-|19|$ &.$/ 999^{* / 5}$ \\
\hline SSI & & & & & &. .1199 \\
\hline
\end{tabular}

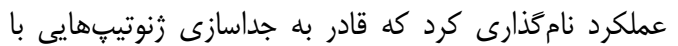

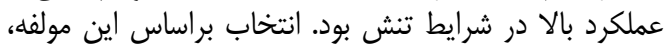

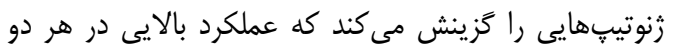

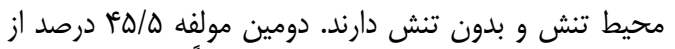

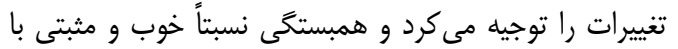

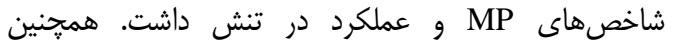
همبستخى متوس و منفى با شاخصهاى
تجزيه بلمولفههاى اصلى براى تعيين رابطه بين

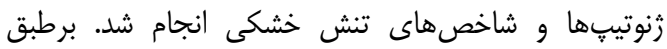

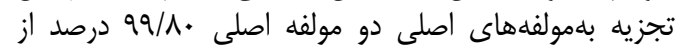

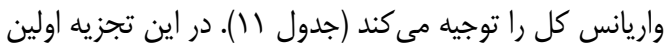
مولفه س/T

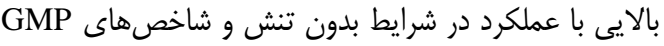

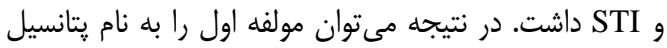


سمت جֶٍ و بالا زنوتيڤهايى كه در محيط تنش خشكى

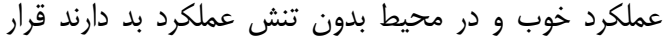

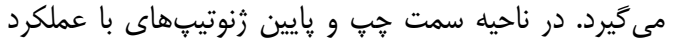

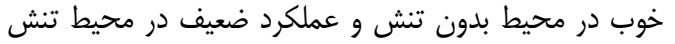

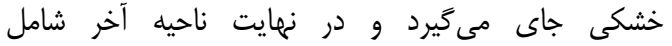

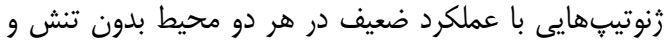

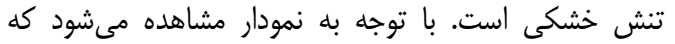

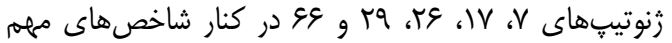
تنش خشكى Yp و Ys و Y Y و

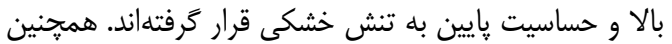

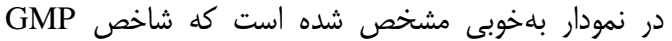

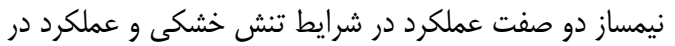

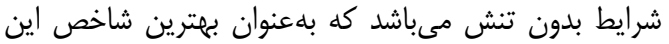

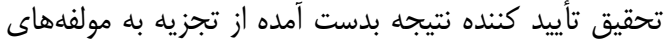

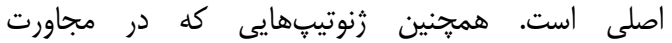

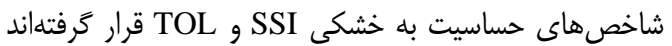

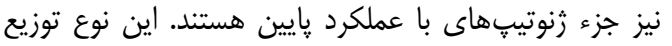

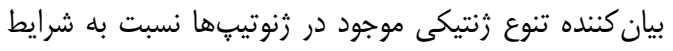

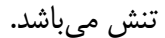

همبستگى منفى بسيار ناجيزى با عملكرد در شرايط آبيارى

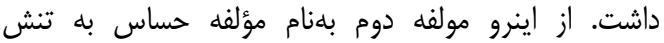

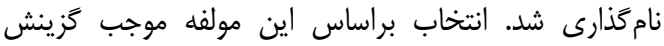

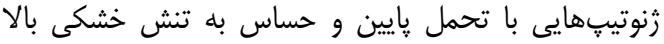

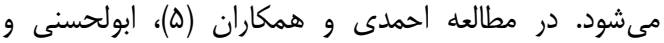

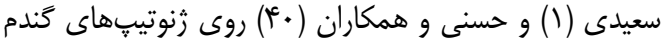

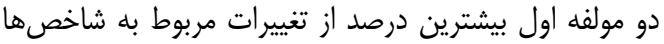

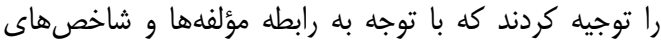

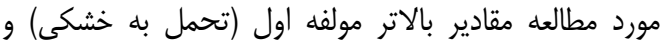

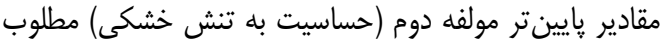

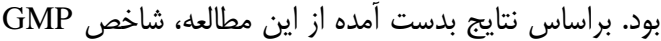

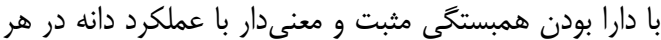

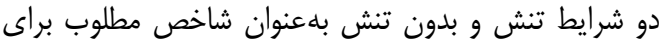

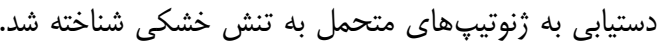

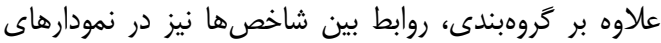

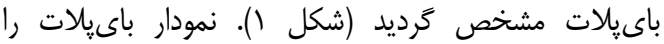

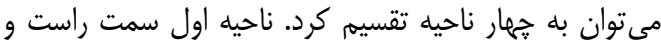

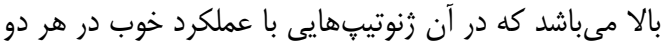

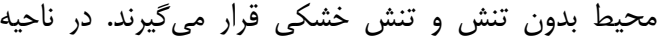

Table 11. Principle Components analysis for drought stress indices

\begin{tabular}{|c|c|c|c|c|c|c|c|c|c|}
\hline & Yp & Ys & TOL & MP & GMP & SSI & STI & Eigenvalue & Proportion \\
\hline $\mathrm{PC} 1$ & $\cdot / D I T$ & $\cdot / \mathrm{T \Lambda}$ & . MFD & - & . & $\cdot / \backslash \wedge \Lambda$ & צמז/. & $\mathrm{r} / \Lambda \cdot 1$ & . \\
\hline $\mathrm{PC} 2$ & r.•|•-. &.$/ 489$ & سו4/.- & . & ./TAM & -.1019 &.$/ 491$ & r/NG & $\cdot / f \Delta \Delta$ \\
\hline
\end{tabular}

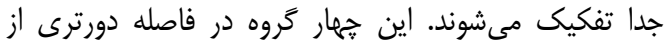

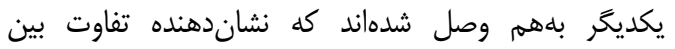

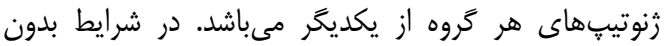

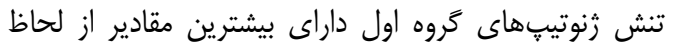

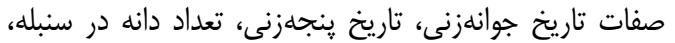

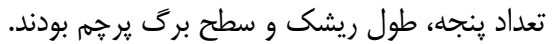

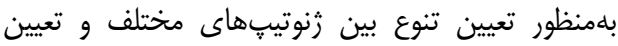

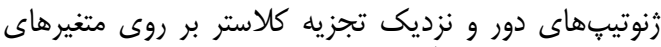

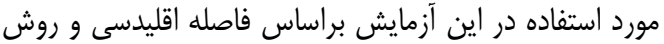

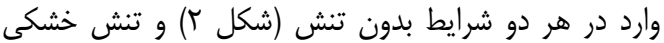

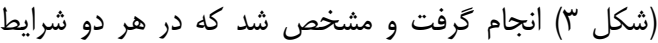

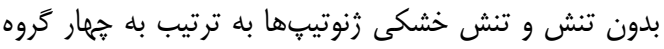

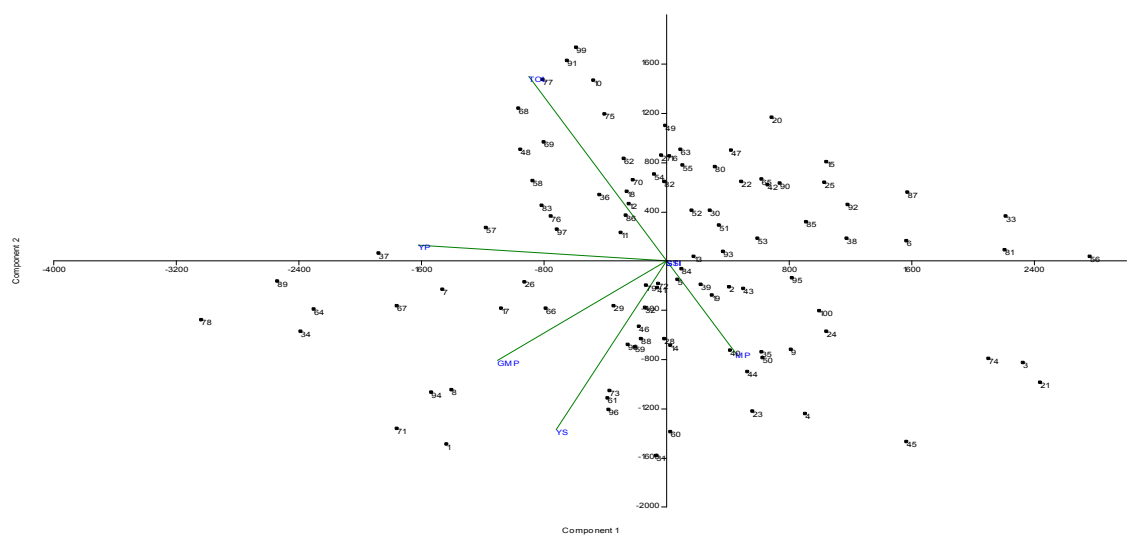

شكل 1 - نمودار باى يلات مولفه اول و مولفه دوم شاخصهاى تحمل به خشكى

Figure 1. Biplot diagram of first and second component of drought stress indices 


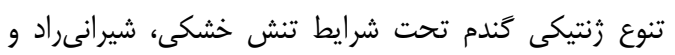

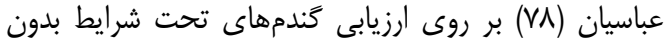

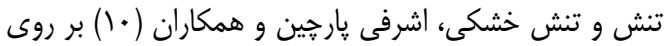

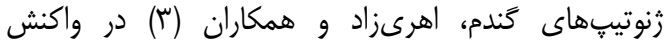

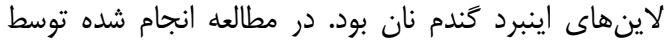

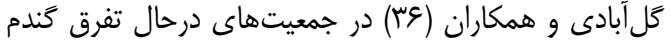

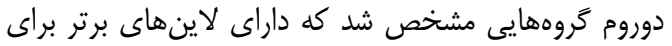

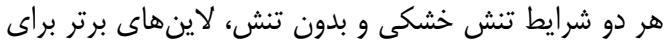

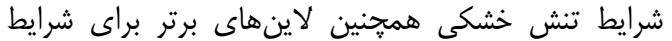

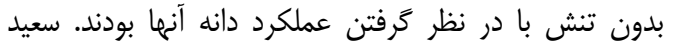

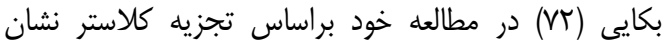

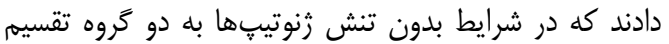

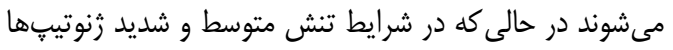

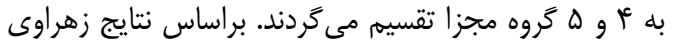

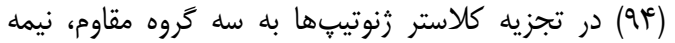
مقاوم و حساس طبقهبندى شدند. تجزيه كلاستر بلهور كلى بلى

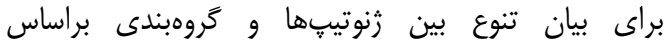

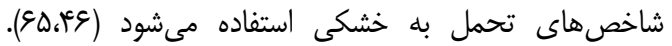

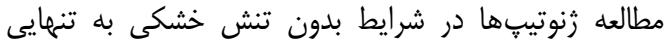

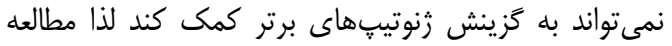

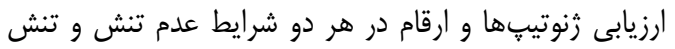

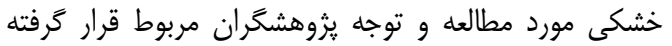

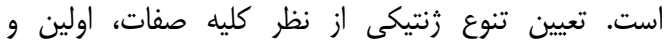
اساسىترين كام در جهت مطالعات تحمل به به خشكى استى مى بـاشد.

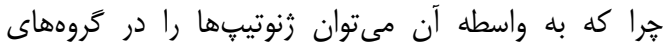

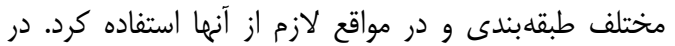

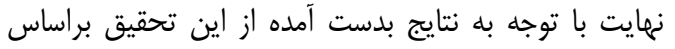

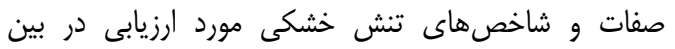

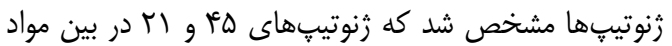

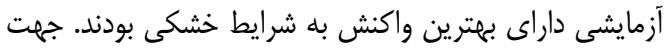

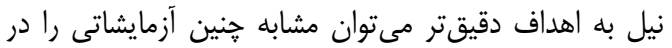

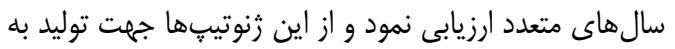

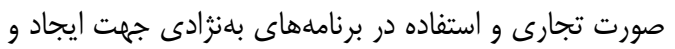
توليد ارقام برتر گَندم نان استفاده كرد.
در گروه دوم زنوتيڤها از لحاظ صفات ارتفاع گياه، طول

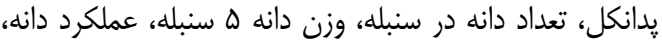

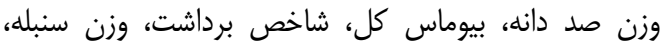
شاخص برداشت و تعداد روز سنبلهدهى تا رسيدگى بـى بيشتر

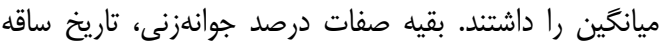

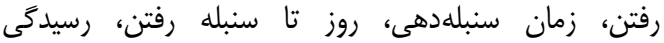

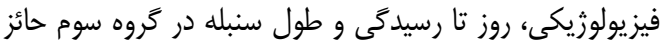

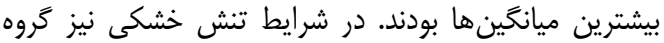

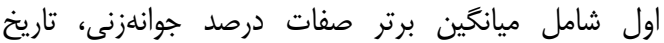

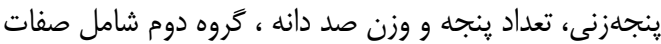

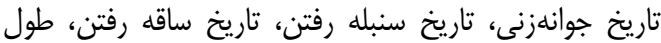
سنبله، طول ريشك، وزن داني، دانه ه سنبله، وزن سن سنبله

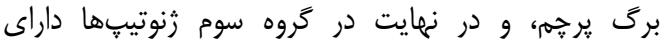

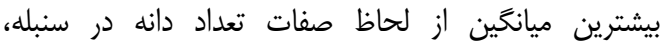

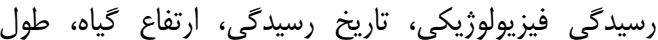

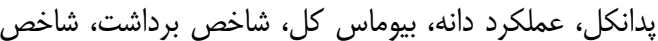

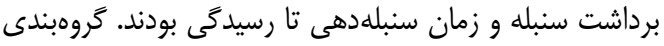

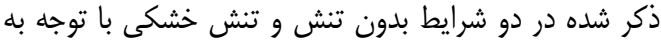

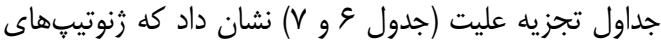

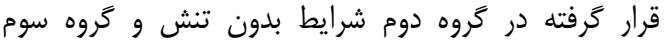

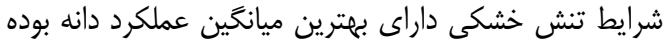

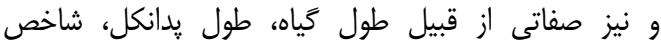

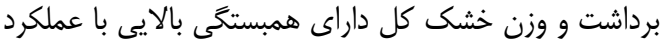

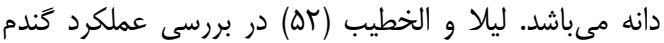
تحت شرايط تنش خشكى سه خوشه راشل را در تجزيه كلا كلاستر

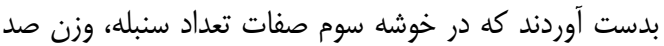

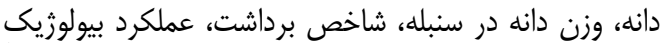

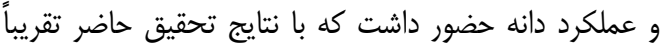

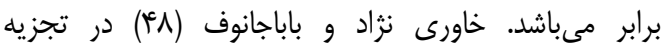

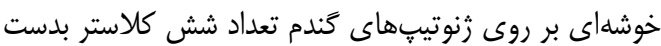

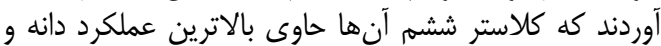

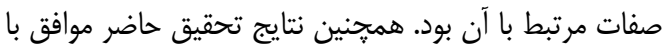

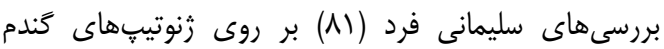

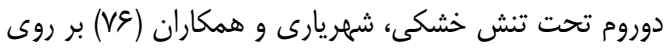




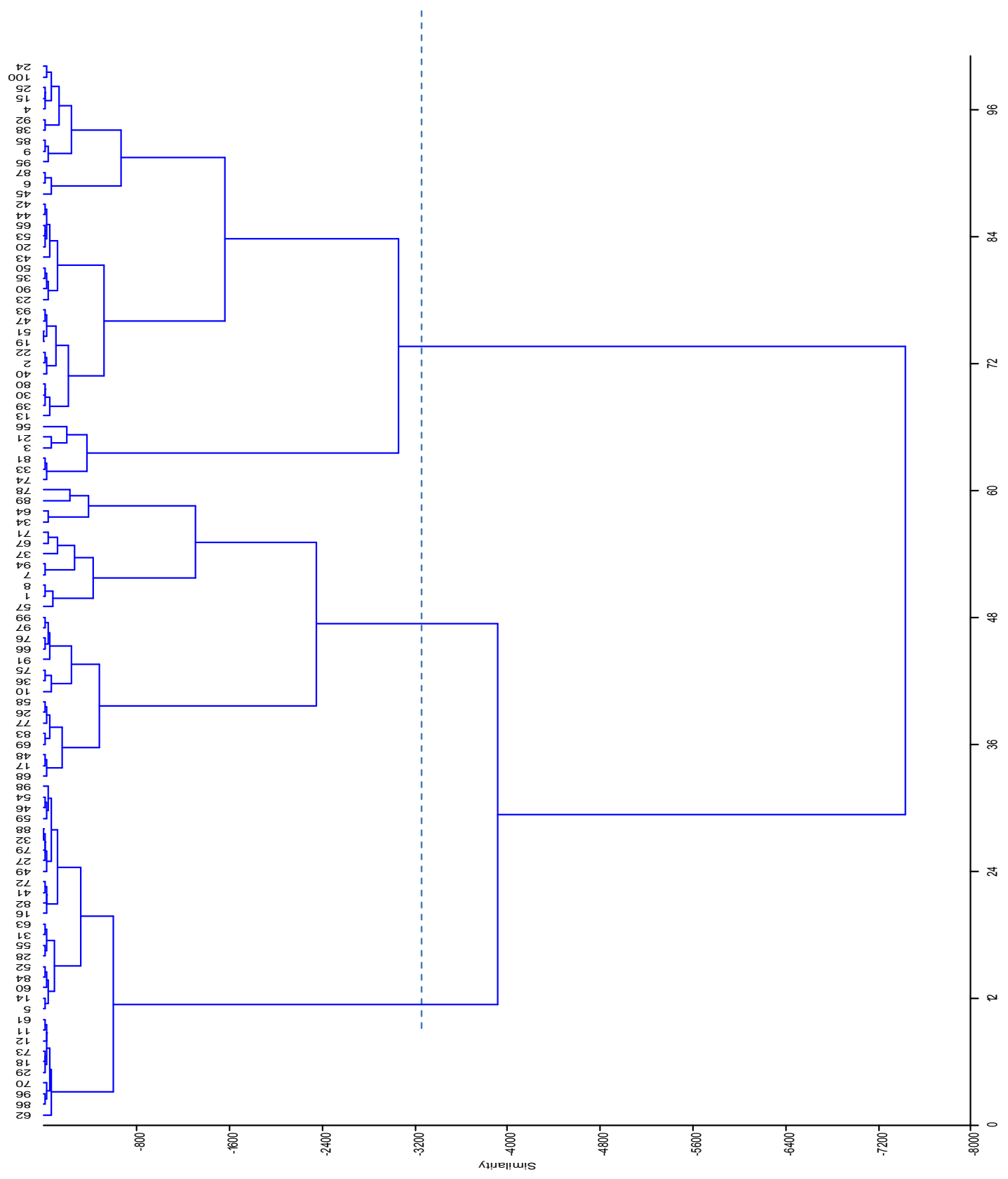

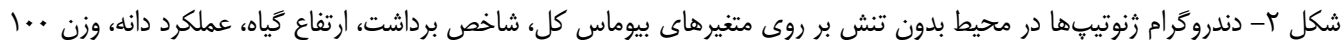

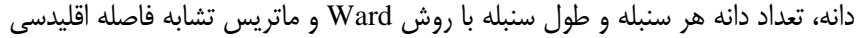

Figure 2. Dendrogram of genotypes under non-stress condition on total biomass, harvest index, plant height, grain yield, 100 grain weight, number of grain in spike by Ward method and Euclidean distance similarity matrix 
1. Abolhasani, K. and G. Saeidi. 2006. Evaluation of drought tolerance in safflower based on tolerant and susceptibility indices to water stress. Water abd Soil Science (Journal of Science and Technology of Agriculture and Natural Researches, 10: 407-418.

2. Aghaee Sarbarzeh, M., M. Rostaee, R. Mohammadi, R. Haghparast and R. Rajabi. 2010. Determination of drought tolerant genotypes in bread wheat. EJCP, 2: 1-23.

3. Aharizad, S., V. Ahmadi and S. Mohammadi. 2011. Bread wheat inbred line responses to drought stress and grouping them. Journal of Crop Ecophysiology (Agriculture Science), 4: 67-78.

4. Ahmadi, G., H. Zeinaly Khane Ghah and M.A. Rostamy. 2000. The study of drought indices and biplot method in eight corn hybrids. Iranian Journal of Agricultural Science, 31: 513-523.

5. Ahmadi, A., M. Saeedi and A. Zalli. 2005. Drought resistance and its relation with yield, leaf area and crop growth rate during reproductive stage in bread wheat genotypes with different breeding backgrounds. Journal of Agricultural Sciences and Natural Resources, 12: 82-90 (In Persian).

6. Ahmadizadeh, M., H. Shahbazi, M. Valizadeh and M. Zaefizadeh. 2011. Genetic diversity of durum wheat landraces using multivariate analysis under normal irrigation and drought stress conditions. African Journal of Agricultural Research, 6: 2294-2302.

7. Alaei, M., M. Zaefizadeh, A. Atamehr, M. Khayatnezhad and Z. Alaei. 2011. Analysis of drought resistance sources from detection function and regression analysis in durum wheat. Advanced Environment Biology, 5: 136-140.

8. Al-Karaki, GA. 2012. Phenological Development-Yield Relationships in Durum Wheat Cultivars under Late-Season High-Temperature Stress in a Semiarid Environment. International Scholarly Research Network, 1-7 pp.

9. Amiri Fahliani, A. and M.T. Assad. 2005. Evaluation of Three Physiological Traits for Selecting Drought Resistant Wheat Genotypes. Journal of Agricultural Science and Technology, 7: 81-87.

10. Ashrafi parchin, R., A. Najaphy, E. Farshadfar and S. Hokmalipour. 2011. Evaluation of wheat genotypes under drought stress based on phonological traits. International Journal of Agriculture and Crop Science, 3: 12-19.

11. Attarbashi, M.R., S. Galeshi, A. Soltani and E. Zinali. 2002. Relationship of phenology and physiologicaltraits with grain yield in wheat under rainfed conditions. Iranian Journal of Agricultural Science, 33: 8-21

12. Azizinya, S., M.R. Bihamta, A.A. Zali, B. Yazdi Samadi and A. Ahmadi. 2005. An evaluation of quantitative traits related to drought resistance in synthetic wheat genotypes in stress and non-stress conditions. Iranian Journal of Agricultural Science, 36: 281-293.

13. Borras, L., G.A. Slafer and M.E. Otegui. 2004. Seed dry weight response to source-sink manipulations in wheat, maize and soybean: a quantitative reappraisal. Field Crops Research, 86: 131-146.

14. Cooper, M., D.E. Byth and D.K. Woodruff. 1994. An investigation of the grain yield adaptation of advanced CIMMYT wheat lines to water stress environments in Queensland I: Crop physiological analysis. Australian Journal of Agricultural Research, 45: 965-984.

15. Damania, A.B. and M.T. Jackson. 1986. An application of factor analysis to morphological data of wheat and barley landraces from the Bheri River Valley, Nepal. Rachis, 5: 25-30.

16. Dastfal, M., V. Barati, Y. Emam, H. Haghighatnia and M. RamezanPour. 2012. Evaluation of grain yield and yield component in wheat genotypes under late drought stress in Darab Zone. Seed and Plant Production Journal, 27: 195-217.

17. Dencic, S., R. Kastori, B. Kobiljski and B. Duggan. 2000. Evaluation of grain yield and its components in wheat cultivars and landraces under near optimal and drought conditions. Euphytica, 113: 43-52.

18. Donald, CM., J. Hamblin and N.C. Brady. 1976. The biological yield and harvest index of cereals as agronomic and plant breeding criteria. In: Advances in Agronomy, 28: 361-405.

19. Drikvand, R., B. Doosty and T. Hosseinpour. 2012. Response of Rain fed Wheat Genotypes to Drought Stress Using Drought Tolerance Indices. Journal of Agricultural Science, 4: 126-131.

20. Ebadi, A., K. Sajed and R. Asgari. 2007. Effects of water deficit on dry matter remobilization and grain filling trend in three spring barley genotypes. Journal of Food Agricultural. Environment, 5: 359-362.

21. Ehdaei, B., G.A. Alloush, M.A. Madore and J.G. Waines. 2006. Genotypic variation for stem reserves and mobilization in wheat. I. Post-anthesis changes in internode dry matter. Crop Science, 46: 735-746.

22. Ehdaie, B. and J.G. Waines. 1996. Genetic variation for contribution of parenthesis assimilates to grain yield spring wheat. Journal of Genetic and Breeding, 50: 47-56.

23. Eskandari, H. and K. Kazemi. 2010. Response of different bread wheat (Triticum aestivum L.) genotypes to post-anthesis water deficit. Notulae Scientia Biologicae, 2: 49-52.

24. Evans, L.T. and I.F. Wardlaw. 1996. In: Zamski E and Schaffer AA (Eds.), Photo assimilate Distribution in Plants and Crops. Marcel Dekker INC, New York, pp: 501-518.

25. Evans, L.T. and R.L. Dunstone. 1970. Some physiological aspects of evaluation in wheat. Australian Journal of Biological Science, 23: 725-741.

26. Ezzat Ahmadi, M., G. NourMohammadi, M. Ghodsi and M. Kafi. 2012. Evaluation of drought tolerance and use of promising bread wheat genotypes stem resources under different water and photosynthesis stress. Iranian Journal of Field Crops Research, 9: 758-769.

27. Fallahi, H.A., J. Alat Jafar Bay and F. Seyyedi. 2012. Evaluation of drought tolerance in Durum wheat genotypes using drought tolerance indices. Seed and Plant Improvement Journal, 27: 15-22.

28. http://www.Fao.org.

29. Farshadfar, E. and J. Sutka. 2003. Multivariate analysis of drought tolerance inwheat substitution lines. Cereal Research Communications, 31: 33-40.

30. Farshadfar, E., M. Farshadfar and F. Farshadfar. 2011. Screening agronomic, physiological and metabolite indicators of drought tolerance in bread wheat (Triticum aestivum L.). American Journal of Scientific Research, 38: 88-96.

31. Fernandez, G.C.J. 1992. Effective selection criteria for assessing plant stress tolerance. In Proceedings of a Symposium. Taiwan, 257-270. 
32. Fischer, R.A. and R. Maurer. 1978. Drought resistance in spring wheat cultivars. I: grain yield response. Australian Journal of Agricultural Research, 29: 897-912.

33. Fischer, R.A. 1973. The effects of water at various stages of development on yield processes in wheat, In: Proceedings of Plant Responses to Climate Factors Symposium. Unesco, Paris, 233-241.

34. Ghaffari, G., M. Toorchi, S. Aharizad and M.R. Shakiba. 2011. Evaluation of traits related to water deficit stress in winter rapeseed cultivars. Universal Journal of Environmental Research and Technology, 1: 338350.

35. Ghamarnia, H. and J.W. Gowing. 2005. Effect of water stress on three wheat cultivars. ICID $21^{\text {st }}$ European Regional Conference. May. Frankfurt (order) and Slubice Germany and Poland, 15-19.

36. Golabadi, M., A. Arzani and M. Maibody. 2006. Assessment of drought tolerance in segregating populations in durum wheat. African Journal of Agriculture Research, 1: 162-171.

37. Golparvar, A.R. 2000. Evaluation of some genotypes of wheat under drought stressed and non-stressed conditions and determination of the most suitable selection criteria in both conditions. MSc. Dissertation, College of Agriculture, University of Tehran, Karaj, 143 pages (in Persian).

38. Hafiz, M., M. Akram, I. Sarfraz, M. Saeed, A. Yar, A. Ali, KA. Sahi and MA. Nadeen. 2004. Drought tolerance studies of wheat genotypes. Pakistan Journal of Biological Science, 71: 90-92.

39. Hamam, K.A. 2008. Increasing yield potential of promising bread wheat lines under drought stress. Journal of Agriculture Biology Science, 4: 842-860.

40. Hasani, S., H. Pirdashti, R. Mesbah and N. Babaian Jolodar. 2007. Evaluation of drought tolerance indices in yield of six cultivars of Virginia tobacco (Nicotiana tabacum L.). Seed and Plant Improvement Journal, 24: 129-144.

41. Hay, R.K. and A.J. Walker. 1994. An introduction to the physiology of crop yield, $571 \mathrm{pp}$.

42. Hohls, T. 2001. Conditions under which selection for mean productivity, tolerance to environmental stress, or stability should be used to improve yield across a range of contrasting environments. Euphytica, 120: 235-245.

43. Johnson, A.M. and D.E. Fowler. 1992. Response of no-till winter wheat to nitrogen fertilization and drought stress. Canadian Journal of Plant Sciences, 72: 1057-1089.

44. Karami, E., M.R. Ghannadha, M.R. Naghavi and M. Mardi. 2005. Identifying of drought tolerant varieties in barley. Iranian Journal of Agriculture Science, 37: 371-379 (In Persian).

45. Karami, E., M.R. Ghannadha, M.R. Naghavi and M. Mardi. 2006. Detection of drought tolerant cultivars in barley. Iranian Journal of Agricultural Sciences, 37: 371-379 (In Persian).

46. Khalili M., MR. Naghavi, AR. Pour Aboughadareh and J. Talebzadeh .2012. Evaluating of Drought Stress Tolerance based on selection indices in spring canola cultivars (Brassica napus L.). JAS, 4:78-85.

47. Khashif, M. and I. Khaliq. 2004. Heritability, correlation and path coefficient analysis for some metric traits in wheat International Journal of Agriculture Biology, 6: 138-142.

48. Khavarinejad, M.S. and A.V. Babajanov. 2011. Identification of relationships of quantitative and morphological traits to spring wheat genotype yields in drought levels of Mazandaran (north of Iran). International Journal of Agricultural Science, 1: 329-339.

49. Khayatnezhad, M., M. Zaefizadeh and R. Gholamain. 2011. Factor analysis of yield and other traits of durum wheat under drought stress and no stress conditions. Plant Echophysiology, 3: 23-27.

50. Khayatnezhad, M., M. Zaefizadeh and R. Gholamain. 2011. Factor analysis of yield and other traits of durum wheat under drought stress and no stress conditions. Plant Echo-physiology, 3: 23-27.

51. Kirigwi, F.M., M. Can Ginkel, R. Trethowan, R.G. Sears, S. Rajaram and G.M. Paulsen. 2004. Evaluation of selection strategies for wheat adaptation across water regimes. Euphytica, 135: 361-371.

52. Leilah, A.A. and S.A. Al-Khateeb. 2005. Statistical analysis of wheat yield under drought conditions. Journal of Arid Environments, 61: 483-496.

53. Lopez, C.G., G.M. Banowetz, C.J. Peterson and W.E. Kronstad. 2003. Dehydrin expression and drought tolerance in seven wheat cultivars. Crop Sci, 43: 577-582.

54. Mardeh. A.S.S., A. Ahmadi, K. Poustini and V. Mohammadi. 2006. Evaluation of drought resistance indices under various environmental conditions, Field Crops Researches, 98: 222-229.

55. Merah, O.J., L. Araus, I. Souyris, M. Nachit, E. Deleens and P. Monneveux. 2001. Carbon isotope discrimination: Potential interest for grain yield improvement in durum wheat. CIHEAM-options Mediterranean's, 299-301.

56. Mi, G., L. Tang, F. Zhang and J. Zhang. 2000. Is nitrogen uptake after anthesis in wheat regulated by sink size? Field Crops Research, 68: 183-190.

57. Moayedi, A.A., A.N. Boyce and S.S. Barakbah. 2010. The performance of durum and bread wheat genotypes associated with yield and yield component under different water deficit conditions. Australian Journal of Basic Applied Science, 4: 106-113.

58. Moghaddam, M., B. Ehdaie and J.G. Waines. 1998. Genetic variation for and inter-relationships among agronomic traits in landraces of bread wheat from southwestern Iran. Journal of Genetics and Breeding, 52: $73-81$.

59. Moghaddasi, L., V. Rashidi and A. Haghighi. 2010. Effect of drought stress on grain yield anf some morphological traits in Durum wheat lines. Journal of Crop Eco-physiology (Agriculture Science), 3: 4153.

60. Mohamed, N.A. 1999. Some statistical procedures for evaluation of the relative contribution for yield components in wheat. Zagazig Journal of Agricultural Research, 26: 281-290.

61. Mohammadi, M., M.R. Ghannadha and A.R. Tale'ee. 2002. Study of the genetic variation within Iranian local bread wheat lines using multivariate techniques. Seed and Plant Improvement Journal, 18: 328-347 (In Persian).

62. Nachit, M.M. and A. Quassou. 1988. Association of yield potential, drought tolerance and stability of yield in T. turgidum var. durum. In:Proceedings of the 7th International wheat symposium. Cambridge, UK, 867870.

63. Naderi, A., E. Majidi-Heravan, A. Hashemi-Dezfuli, A.M. Rezaie and G. NourMohamadi. 2000. Efficiency analysis of indices for tolerance to environmentalstresses in field crops and introduction of a new index. Seed and Plant, 15: 390-402 (In Persian). 
64. Naghavi, M., A. Shahbazi and A. Talaeei. 2002. Study of diversity of agronomy and morphological traits of genetics resources of durum wheat. Journal of Agronomy Science. Iran, 2: 81-88.

65. Naghavi, M.R., A.R. Pour-Aboughadareh and M. Khalili. 2013. Evaluation of drought tolerance indices for screening some of corn (Zea mays L.) cultivars under environmental conditions. Notulae Scientia Biologicae, 5: 388-393.

66. Oosterhius, D.M. and P.M. Carwtright. 1983. Spike differentiation and floret survival in semi dwarf spring wheat as affected by water stress and photoperiod. Crop Science, 23: 711-717.

67. Pheloung, P.C. and K.H.M. Siddique. 1991. Contribution of stem reserves to grain yield inwheat cultivars. Australian Journal of Plant Physiol, 18: 53-64.

68. Richard, R.A. 1996. Defining selection criteria to improve yield under drought. Plant Growth Regulation, 20: 157-166.

69. Richards, R.A., A.G. Condon and G.J. Rebetzke. 2001. Traits to improve yield in dry environments. Application of Physiology in wheat breading, M.P. Reynolds et al. (Eds.), CIMMYT, 88-110 pp.

70. Roostaee, M., D. Sadeghzadeh and Y. Arshad. 2003. Study of relationship of wheat grain yield-affecting traits by factor analysis under rain-fed conditions. Agriculture Knowledge, 13 pp (In Persian).

71. Royo, C., M. Abaza, R. Blanco and L.F. Garcia Del Moral. 2000. Triticale grain growth and morphometry as affected by drought stress, late sowing and simulated drought stress. Australian Journal of Plant Physiology, 27: 1051-1059.

72. Sajad-Bokaei, A., H. Babaei, D. Habibi, S. Javidfar and A. Mohammadi. 2008. Evaluation of different soybean (Glycine max L.) genotypes under drought stress conditions. Journal of Agronomy and Plant Breeding, 4: $28-38$.

73. Sanjari Pireivatlou, A. and A. Yazdansepas. 2008. Evaluation of Wheat (Triticum aestivum L.) Genotypes under Pre- and Post-anthesis Drought Stress Conditions. Journal of Agriculture Science Technology, 10: 109-121.

74. Schneider, A.D., J.T. Musick and D.A. Dusek. 1969. Efficient wheat irrigation with limited water. Transaction of the ASAE, 12: 23-26.

75. Semeena, S. and L. Singh. 2001. Studies on path co-efficient analysis of harvest index and its related traits in wheat. Indian Journal of Agriculture Science, 35: 127-129.

76. Shahryari, R., B. Mahfoozi, V. Mollasadeghi and M. Khayatnezhad. 2011. Genetic diversity in bred wheat for phonological and morphological traits under terminal drought stress condition. Advanced Environment Biology, 5: 169-172.

77. Shimshi, D., M.L. Mayoral and D. Atsmon. 1982. Responses to water stress in wheat and related wild species. Crop Science, 22: 123-128.

78. Shirani Rad, A.H. and A. Abbasian. 2011. Evaluation of drought tolerance in rapeseed genotypes under non stress and drought stress conditions. Notulae Botanicae Horti Agrobotanici Cluj-Napoca, 39: 164-171.

79. Siani, H.S. and D. Aspinall. 1981. Effects of water deficit on sporogenesis in wheat. Annals of Botany, 43: 623-633.

80. Sio-se Mardeh, A., A. Ahmadi, K. Poustini and V. Mohammadi. 2006. Evaluation of drought resistance indices under various environmental conditions. Field Crop Researches, 98: 222-229.

81. Soleymanifard, A., R. Naseri and M. Moradi. 2012. The study genetic variation and factor analysis for agronomic traits of Durum wheat genotypes using cluster analysis and path analysis under drought stress condition in western of Iran. Journal of Applied Basic Science, 3: 479-485.

82. Solomon, KF. and MT. Labuschagne. 2004. Inheritance of evapotranspiration and transpiration efficiencies in diallel $\mathrm{F}$ hybrids of durum wheat (Triticum turgidum L. var. durum). Euphytica, 136: 69-79.

83. Subhani, G.M. and M.A. Chowhdry. 2000. Correlation and path coefficient analysis in bread wheat under drought stress and normal conditions. Pakistan Journal of Biological Sciences, 3: 72-77.

84. Sundari, T., S. Tohari and W. Mangoendidjojo. 2005. Yield performance and tolerance of mungbeam genotypes to shading. Ilmu. Pertanian, 12: 12-19.

85. Talebi, R., F. Fayyaz and A.M. Naji. 2010. Genetic variation and interrelationships of agronomic characteristics in durum wheat under two constructing water regimes. Brazilian Archives of Biology and Technology, 53: 785-791.

86. Tousi Mojarad, M., M.R. Ghanadha, M. Khodarahimi and S. Shahabi. 2005. Factor analysis for grain yield and other traits of wheat. Agronomy Journal, 67: 9-16.

87. Trethowan, R.M., M. Van Ginkel and S. Rajram. 2002. Progress in breeding wheat for yield and adaptation in global drought affected environments. Crop Science, 42: 1441-1446.

88. Vijendra Das, L.D. 2000. Problems facing plant breeding. CBS Publishers and Distributors. New Delhi, India, 242 pages.

89. Wardlaw, I.F. 1971. The early stages of grain development in wheat. Response to water stress in a single variety. Australian Journal of Biology Science, 24: 1047-1055.

90. Warrington, I.J., R.I. Dunstone and L.M. Green. 1997. Temperature effects at three development stages on the yield of the water ear. Australian Journal of Agricultural Research, 28: 11-27.

91. Yadav, R.S., C.T. Hash, F.R. Bidinger and C.J. Howarth. 1999. Identification and utilization of quantitative trait loci to improve terminal drought tolerance in pearl millet (Pennisetum Glaucum L. R. Br.) CIMMYT. Workshop on Molecular Approaches for the genetic improvement of cereals for stable production in waterlimited Environments, 108-113.

92. Yang, J., J. Zhang, Z. Huang, Q. Zhu and L. Wang. 2000. Remobilization of carbon reserves is improved by controlled soil drying during grain filling of wheat. Crop Science, 40: 1645-1655.

93. Zaeifizadeh, M., M. Khayatnezhad, M. Ghasemi, J. Azimi and M. Vahabzadeh. 2011. Path Analysis of Yield and Yield Components in Synthetic Bread Wheat (Triticum aestivum L.) Genotypes. Advaced Environment Biology, 5: 98-103.

94. Zahravi, M. 2009. Evaluation of Genotypes of Wild Barley (Hordeum spontaneum) Based on Drought Tolerance Indices. Seed and Plant Improvement Journal, 25: 533-549. 


\title{
Evaluation of Durum Wheat Genotypes under Normal Irrigation and Drought Stress Conditions
}

\author{
Mohammad Reza Bihamta ${ }^{1}$, Mehdi Shirkavand ${ }^{2}$, Javad Hasanpour ${ }^{3}$ and Amin Afzalifar ${ }^{4}$ \\ 1- Professor, Department of Agronomy and Plant Breeding, University of Tehran, \\ (Coresponding Author: mrghanad@ut.ac.ir) \\ 2- M.Sc. Student, Islamic Azad University, Science and Research Branch \\ 3- Assistant Professor, Varamin Agronomy Research Center \\ 4- PhD Student, Department of Agricultural Science, Payam Noor University, Tehran \\ Received: June 20, $2016 \quad$ Accepted: September 25, 2016
}

\begin{abstract}
The use of high yielding and resistant to drought stress cultivars in crops is one of the proper way to incorporation other water deficit management methods. This study in order to evaluate grain yield and morphological traits, an experiment was conducted with 100 genotypes of durum wheat which were planted based on lattice design with three replications under normal and drought stress conditions at the Agricultural Research Station in Varamin. The results showed that total dry matter have a highest positive direct effect on grain yield, whereas, grain yield filling period had the lowest effect and grain yield filling period via harvest index had the positive indirect effect on yield. Under stress condition, peduncle length had the highest positive direct effect on grain yield whereas, spike number, plant length, total dry matter, and date from germination to maturity had negative and direct effect. Factor analysis explained 80.5 and 83.3 of the total variation under normal and drought conditions, respectively. To estimate better grain yield, drought tolerance indices were determined and genotypes 3, 45 and 21 were identified with the lowest value of the indices MP, GMP and STI that were for drought condition while genotypes $45,21,71,34,78$ had the highest value of the mentioned indices and were suitable for both conditions. Cluster analysis grouped genotypes into four clusters in both conditions which confirmed the results of stepwise regression as well as path analysis that could separate genotypes with high grain yield from others as well as traits affecting yield compared to other traits.
\end{abstract}

Keywords: Cluster analysis, Drought tolerance, Drought tolerance indices, Durum wheat, Path Analysis 